\title{
A CARLESON TYPE THEOREM FOR A CANTOR GROUP MODEL OF THE SCATTERING TRANSFORM
}

\author{
CAMIL MUSCALU, TERENCE TAO, AND CHRISTOPH THIELE
}

\begin{abstract}
We consider a basic $d$-adic model for the scattering transform on the line. We prove $L^{2}$ bounds for this scattering transform and a weak $L^{2}$ bound for a Carleson type maximal operator (Theorem 1.4). The latter implies boundedness of $d$-adic models of generalized eigenfunctions of Dirac type operators with potential in $L^{2}(\mathbb{R})$. We show that this result cannot be obtained by estimating the terms in the natural multilinear expansion of the scattering transform (Proposition 5.1).
\end{abstract}

\section{INTRODUCTION}

It is widely understood that scattering transforms are non-linear variants of the one dimensional Fourier transform. Thus scattering transforms give nonlinear Fourier transforms of scalar or more generally matrix valued potentials $F(x)$. For harmonic analysts this suggests to study the basic a priori estimates in Fourier analysis (such as for example Hausdorff Young inequalitites or estimates for the Carleson operator) in the case of the scattering transforms. This naturally leads to the study of the nonlinear Fourier transform for rough and slowly decaying potentials. Beals and Coifman [2] study in detail the case when the potential is (generic) in $L^{1}$ or in weighted spaces $L^{1} \cap L^{2}$ with weights of the form $(1+|x|)^{m}$ for suitable $m$. More recently, Christ and Kiselev [6], [7] have proven analogues of the Hausdorff Young inequality and a maximal Hausdorff Young inequality for a scattering transform. This is an estimate for potentials in $L^{p}$. Their result implies boundedness of eigenfunctions of one dimensional Schrödinger operators with potential in $L^{p}, 1<p<2$ for almost all positive energies. By an extension by Simon of a theorem of Sch'nol 13 this implies that the absolutely continuous spectrum of the Schrödinger operator is supported on the entire positive half axis, see also [14] page 501. This implication was one of the motivations of Christ and Kiselev to study the maximal Hausdorff Young inequalities for the scattering transform. We propose to study the analogue of Carleson's theorem [5] or the sharper form by Hunt [9], see also [10] for a recent proof, for scattering transforms. This amounts to an $L^{2}$ endpoint of the results by Christ and Kiselev and would give boundedness of eigenfunctions of Schrödinger operators with potential in $L^{2}$. The question of absolutely continuous spectrum for potentials in $L^{2}$ has been settled to the affirmative by Deift and Killip [8], but this is a weaker statement than the conjectured boundedness of eigenfunctions.

Currently we are not able to decide whether the analogue of Carleson's theorem as stated below is true or false. The purpose of this article is to study a $d$-adic model for this problem and prove a positive result for this model. 
We restrict attention to one of the easiest cases of the scattering transform. Thus consider the special AKNS-ZS system (named after [1] and [18]):

$$
\frac{d f}{d x}=k J f(x)+q(x) f(x)
$$

for the unknown function $f: \mathbb{R} \rightarrow \mathbb{C}^{2}$ where $k \in \mathbb{C}$ is a spectral parameter,

$$
J=\left(\begin{array}{cc}
-i & 0 \\
0 & i
\end{array}\right), q(x)=\left(\begin{array}{cc}
0 & F(x) \\
F(x) & 0
\end{array}\right) .
$$

This can be read as eigenfunction equation for Dirac operators on the real line. More generally one can write the eigenfunction equation for Schrödinger operators on the real line in the framework of AKNS-ZS systems. This links to the work of Christ and Kiselev, but we shall not elaborate on this generalization. We remark that Conjecture 1.3 (as well as the other conjectures formulated below) would imply boundedness of solutions to (1) for almost every $k \in \mathbb{R}$.

We shall assume that $F$ is locally integrable and for simplicity compactly supported. Writing $a(x) \exp (-i k x)$ and $b(x) \exp (i k x)$ for the two components of $f$ and assuming $k$ is real we obtain the following equivalent ordinary differential equation

$$
G^{\prime}=W G
$$

where

$$
G=\left(\begin{array}{cc}
a & b \\
\bar{b} & \bar{a}
\end{array}\right), \quad W(x)=\left(\begin{array}{cc}
0 & F(x) \exp (2 i k x) \\
\bar{F}(x) \exp (-2 i k x) & 0
\end{array}\right) .
$$

Since $F$ is compactly supported, equation (2) forces $G$ to be constant near $-\infty$ and near $\infty$. Let $G(-\infty)$ and $G(\infty)$ denote these constant values. Imposing the initial condition $G(-\infty)=$ id, then standard existence theorems give a unique absolutely continuous solution satisfying (2) almost everywhere. Thus we can define $G(\infty)$ to be the scattering transform of the potential $F$ at the spectral value $k$.

We have implicitly used that the differential equation (2) forces $G$ to remain in the form stated in (3), if it is initially of that form. It also forces $G$ to have constant determinant, which for our chosen initial condition is equal to 1 . In other words, $G$ takes values in the Lie group $S U(1,1)$, see also the discussion in Section 2 .

To prove a priori estimates for the scattering transform, we need a notion of size for the matrices $G$. A natural size appearing in the $L^{2}$ theory of this scattering transform is $\sqrt{\log |a|}$ where $a$ is the upper left entry of the matrix $G$. Observe that this quantity is positive since $|a|^{2}-|b|^{2}=1$.

The following are known analogues of standard estimates for the Fourier transform: Recall that $a(\infty)$ and $a(x)$ for given $x$ are functions in the parameter $k$ which we have suppressed in the notation.

Theorem 1.1. Riemann - Lebesgue estimate

$$
\|\sqrt{\log |a(\infty)|}\|_{\infty} \leq C\|F\|_{1}
$$

Hausdorff Young estimate $(1<p<2)$

$$
\|\sqrt{\log |a(\infty)|}\|_{p^{\prime}} \leq C_{p}\|F\|_{p}
$$


Plancherel identity

$$
\|\sqrt{\log |a(\infty)|}\|_{2}=\frac{\pi}{2}\|F\|_{2}
$$

Maximal Riemann Lebesgue estimate

$$
\left\|\sup _{x} \sqrt{\log |a(x)|}\right\|_{L^{\infty}(k)} \leq C\|F\|_{1}
$$

Maximal Hausdorff Young estimate $(1<p<2)$

$$
\left\|\sup _{x} \sqrt{\log |a(x)|}\right\|_{L^{p^{\prime}(k)}} \leq C_{p}\|F\|_{p}
$$

The Riemann Lebesgue and maximal Riemann Lebesgue estimates follow easily from Gronwall's inequality, i.e., from applying operator norms to (2) and integrating the inequality

$$
\|G\|^{\prime} /\|G\| \leq\|W\|
$$

Then one uses $\sqrt{\log |a|} \sim \log \|G\|$ for small values of $a$ and $\log |a| \sim \log \|G\|$ for large values of $a$. We remark that in the $L^{1}$ theory one may view $\log \|G\|$ as the more natural measure of the size of $G$ than $\sqrt{\log |a|}$.

The Hausdorff Young and maximal Hausdorff Young inequalities follow by the work of Christ and Kiselev [6], [7]. The Plancherel identity is a well known scattering identity.

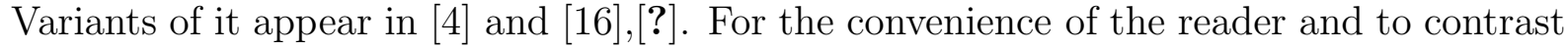
it to our results in the $d$-adic model we will sketch a proof in the appendix. Interestingly, while Plancherel gives the $L^{2}$ endpoint of the Hausdorff Young inequality, we do not known whether the constant $C_{p}$ in the Hausdorff Young inequality can be chosen uniformly as $p$ tends to 2 .

The maximal version of Plancherel, which amounts to a scattering variant of Carleson's theorem, is not known. We state it as a conjecture

Conjecture 1.2. Carleson-Hunt estimate

$$
\left\|\sup _{x} \sqrt{\log |a(x)|}\right\|_{L^{2}(k)} \leq C\|F\|_{2}
$$

A more modest conjecture is

Conjecture 1.3. Weak type Carleson estimate

$$
\left|\left\{k: \sup _{x} \sqrt{\log |a(x)|}>\lambda\right\}\right| \leq C \lambda^{-2}\|F\|_{2}^{2} .
$$

Even more modestly one could conjecture that the function $\sup _{x} \sqrt{\log |a(x)|}$ is finite almost everywhere for $F$ in $L^{2}(\mathbb{R})$. To make $G$ well defined for this last conjecture which is formulated in terms of the scattering transform for arbitrary $F \in L^{2}(\mathbb{R})$ one may replace the initial condition $G(-\infty)=$ id by $G(0)=$ id.

The main purpose of the current article is to give some supporting evidence at least for Conjecture 1.3 by proving a variant of it in a $d$-adic model. The $d$-adic model is obtained by replacing the exponential functions in (3), which are the characters on $\mathbb{R}$, by characters of an infinite product of copies of $\mathbf{Z}(d)$ for some integer $d>1$. We call these groups Cantor groups. 
From now on, $x$ and $k$ will denote non-negative real numbers. For almost all such numbers, we have unique expansions with base $d$ :

$$
k=\sum_{n \in \mathbf{Z}} k_{n} d^{n}, \quad x=\sum_{n \in \mathbf{Z}} x_{n} d^{n}
$$

where $k_{n}$ and $x_{n}$ take values in $0,1, \ldots, d-1$ and they are zero for sufficiently large positive index $n$. Indeed, we shall make these expansions unique for all $x$ and $k$ by requiring each of them to have only finitely many non-zero entries whenever possible.

Then we define a character function $w$ on $\mathbb{R}_{0}^{+} \times \mathbb{R}_{0}^{+}$as

$$
w(k, x)=\gamma^{\sum_{n \in \mathbf{Z}} k_{n} x_{-1-n}},
$$

where $\gamma \in \mathbb{C}$ is some fixed primitive $d$-th root of unity. The exact choice of $\gamma$ is not important. Observe that the formally infinite sum in the exponent in (5) has only finitely many non-zero summands.

Let $F \in L^{2}\left(\mathbb{R}^{+}\right)$. For every parameter $k$ we consider the following initial value problem:

$$
\begin{gathered}
\partial_{x} G(k, x)=W(k, x) G(k, x) \\
G(k, 0)=\mathrm{id}
\end{gathered}
$$

where

$$
W(k, x)=\left(\begin{array}{cc}
0 & F(x) w(k, x) \\
F(x) w(k, x) & 0
\end{array}\right)
$$

By standard ODE theory this initial value problem has a unique absolutely continuous solution satisfying the ODE almost everywhere.

We denote again by $a(k, x)$ the upper left entry of $G(k, x)$. The main theorem of this article is the following

Theorem 1.4. Let $d>1$ and $F \in L^{2}\left(\mathbb{R}^{+}\right)$, and let $G$ be defined by (G). Then for almost all $k \in \mathbb{R}^{+}$the limit

$$
G(k, \infty)=\lim _{x \rightarrow \infty} G(k, x)
$$

exists and satisfies the estimate

$$
\int_{0}^{\infty} \log |a(k, \infty)| d k \leq C \int_{0}^{\infty}|F(x)|^{2} d x
$$

Moreover,

$$
\left|\left\{k: \sup _{x}|a(k, x)|>\lambda\right\}\right| \leq C \lambda^{-1}\|F\|_{2}^{2}
$$

for all $\lambda>0$. Here as well as in (9) the constant $C$ may grow polynomially in $d$ but is independent of $F$ and $\lambda$.

If $F$ is real valued, then a special situation occurs in Theorem 1.4 for $d=2$ : the matrices $W(k, x)$ then are real valued and commute for different values of $x$. By simultaneously diagonalizing all these matrices one can decouple the two equations and obtain an ODE of the form $\tilde{G}^{\prime}=V \tilde{G}$ with

$$
V(k, x)=\left(\begin{array}{cc}
F(x) w(k, x) & 0 \\
0 & -F(x) w(k, x)
\end{array}\right) .
$$


The solution at $+\infty$ of the corresponding initial value problem is

$$
V(k, x)=\left(\begin{array}{cc}
\exp (\hat{F}(x)) & 0 \\
0 & \exp (-\hat{F}(x))
\end{array}\right)
$$

where $\hat{F}$ denotes the Walsh-Fourier transform (the Fourier transform with respect to the Cantor group with $d=2$ ). In this special case Theorem 1.4 follows simply from the known Plancherel identity and Carleson's theorem for the Walsh Fourier transform [3]. This example shows nicely the connection of scattering transforms and the Fourier transform.

We will outline the proof of (8) in Section 2. The proof is based on certain swapping inequalities, which are discussed in detail in Section 3. The proof of these inequalities seems to be a genuinely new ingredient in the $d$-adic model as compared to the theory of the linear Fourier transform. In Section 4 we prove (9), which then easily implies (7).

Initially the authors had attempted to use multilinear expansions of the solutions to (6) to prove Conjecture 1.3 in the way Christ and Kiselev prove their results for $p<2$. However, as was observed in [11], the terms in this expansion do not satisfy reasonable bounds for $F \in L^{2}(\mathbb{R})$. Since the purpose of the current article is to compare the $d$-adic to the continuous case, we prove a result (Proposition 5.1) in Section 5 which shows that the multilinear terms in the $d$-adic setting are equally badly behaved. This is the second new result of this article.

In the appendix (Section 6) we sketch a proof of the Plancherel identity (4) in Theorem 1.1. We only know a proof of this identity using complex contour integration. This proof seems to not have the same flexibility as the proof in the $d$-adic case which decomposes the scattering transform into its elementary pieces. This in a sense is the main reason why at this point we are unable to prove Carleson's theorem for the continuous scattering transform.

The first author was supported by NSF grant DMS 0100796. The second author is a Clay Prize Fellow and is supported by a grant from the Packard Foundations. The third author was supported by a Sloan Fellowship and by NSF grants DMS 9985572 and DMS 9970469.

\section{Proof of the Plancherel inequality (8)}

First we consider the case of compactly supported $F$. Thus, for fixed $k, G(k, x)$ becomes constant for large $x$ and the existence of the limit $G(k, \infty)$ is not in question.

Recall that $S U(1,1)$ is the Lie group of all complex $2 \times 2$ matrices of the form

$$
\left(\begin{array}{ll}
a & b \\
\bar{b} & \bar{a}
\end{array}\right)
$$

with determinant $|a|^{2}-|b|^{2}=1$. This group is isomorphic to $S L_{2}(\mathbb{R})$. Observe that $W(k, x)$ is an element of the Lie algebra of $S U(1,1)$, and thus the solution to the initial value problem (6), which is well known to exist as an absolutely continuous function, takes values in $S U(1,1)$. Of course one can verify directly by an elementary calculation that the solution to (6) has the form (10) and determinant 1 for all $x$, which is all we need from this brief discussion of Lie groups. 
The following is an easy observation about breaking the ODE (6) into pieces along the $x$ variable. For any interval $\omega \subset \mathbb{R}_{0}^{+}$define the localized system

$$
\begin{gathered}
\partial_{x} G_{\omega}(k, x)=W_{\omega}(k, x) G_{\omega}(k, x) \\
G_{\omega}(k, 0)=\mathrm{id},
\end{gathered}
$$

where

$$
W_{\omega}(k, x)=\left(\begin{array}{cc}
0 & F(x) 1_{\omega}(x) w(k, x) \\
F(x) 1_{\omega}(x) w(k, x) & 0
\end{array}\right)
$$

Lemma 2.1. Let $\omega_{1}, \omega_{2}, \ldots, \omega_{n}$ be adjacent intervals in ascending order, and let the union of these intervals be the interval $\omega$. Then we have for all $k \geq 0$ :

$$
G_{\omega}(k, \infty)=\prod_{j=n}^{1} G_{\omega_{j}}(k, \infty)=G_{\omega_{n}}(k, \infty) \ldots G_{\omega_{2}}(k, \infty) G_{\omega_{1}}(k, \infty)
$$

Proof: By induction the lemma follows from the special case for two adjacent intervals $\omega_{1}$ and $\omega_{2}$. Fix $k$. It is easy to check that the absolutely continuous function

$$
G_{\omega_{2}}(k, x) G_{\omega_{1}}(k, x)
$$

satisfies the differential equation for $G_{\omega}(k, x)$ almost everywhere. This follows easily from letting $x_{0}$ be the point separating $\omega_{1}$ and $\omega_{2}$ and considering $x<x_{0}$ and $x>x_{0}$ separately. Since (12) also satisfies the correct initial condition, this proves the lemma.

Next, we claim that if $\omega$ is a $d$-adic interval, that means an interval of the form

$$
\left[d^{\kappa} n, d^{\kappa}(n+1)\right)
$$

with integers $\kappa$ and $n \geq 0$, then $G_{\omega}(k, x)$ does not change much as $k$ varies inside a $d$-adic interval of reciprocal length $d^{-\kappa}$.

To make this claim precise, we define a tile to be a rectangle $p=I \times \omega$ of the form

$$
\left[d^{\kappa} n, d^{\kappa}(n+1)\right) \times\left[d^{-\kappa} l, d^{-\kappa}(l+1)\right)
$$

with integers $\kappa, n, l$ such that $l, n \geq 0$.

Lemma 2.2. Let $I \times \omega$ be a tile. Let $k_{0}$ be the left endpoint of $I$ and let $k$ be any point in $I$. Then there is an integer $j=j(k)$ independent of $x$ such that if

$$
G_{\omega}\left(k_{0}, x\right)=\left(\begin{array}{cc}
a & b \\
\bar{b} & \bar{a}
\end{array}\right)
$$

then

$$
G_{\omega}(k, x)=\left(\begin{array}{cc}
\frac{a}{\gamma^{j} b} & \gamma^{j} b
\end{array}\right)
$$

In particular, the first entry a of $G_{\omega}(k, x)$ is independent of $k$ as long as $k \in I$. 
Proof: Assume the length of $I$ is $d^{\kappa}$. Since $G_{\omega}$ is constant outside $\omega$, it suffices to show the claim for $x \in \omega$. We split $w(k, x)$ into two factors as follows:

$$
w(k, x)=\left(\gamma^{\sum_{\nu<\kappa} k_{\nu} x_{-1-\nu}}\right)\left(\gamma^{\sum_{\nu \geq \kappa} k_{\nu} x_{-1-\nu}}\right) .
$$

Observe that if $x$ varies in $\omega$, then the first factor in this splitting does not change. Likewise, the second factor is constant for $k \in I$. Thus there is a $j$ depending on $k \in I$ such that

$$
w(k, x) 1_{\omega}(x)=\gamma^{j} w\left(k_{0}, x\right) 1_{\omega}(x) .
$$

Now let $\Gamma$ be the constant matrix

$$
\Gamma=\left(\begin{array}{cc}
\gamma^{j} & 0 \\
0 & 1
\end{array}\right)
$$

Then

$$
W_{\omega}(k, x)=\Gamma W_{\omega}\left(k_{0}, x\right) \Gamma^{-1} .
$$

By conjugating the initial value problem (11) by $\Gamma$ we observe that

$$
G_{\omega}(k, x)=\Gamma G_{\omega}\left(k_{0}, x\right) \Gamma^{-1} .
$$

This proves the lemma.

Motivated by this lemma we shall define for a tile $p=I \times \omega$ :

$$
G_{p}=G_{\omega}\left(k_{0}, \infty\right)
$$

where $k_{0}$ is the left endpoint of $I$. Next, we shall investigate the relation of the matrices $G_{p}$ for nearby tiles $p$. Here we mean by nearby tiles that the tiles are contained in a given $d$-adic rectangle of area $d$.

Define a multitile to be a rectangle $P=I \times \omega$ of the form

$$
\left[d^{\kappa} n, d^{\kappa}(n+1)\right) \times\left[d^{1-\kappa} l, d^{1-\kappa}(l+1)\right)
$$

with integers $\kappa, n, l$ and $l, n \geq 0$. There are $d$ tiles $p_{j}, j=0, \ldots, d-1$ contained in $P$ of the form $I \times \omega_{j}$. We shall always assume the $\omega_{j}$ are ordered in ascending order. We call these tiles the horizontal subtiles of $P$. Moreover, there are $d$ tiles $q_{j}, j=0, \ldots, d-1$ contained in $P$ of the form $I_{j} \times \omega$. We shall again assume the $I_{j}$ are ordered in ascending order, and we call these tiles the vertical subtiles of $P$.
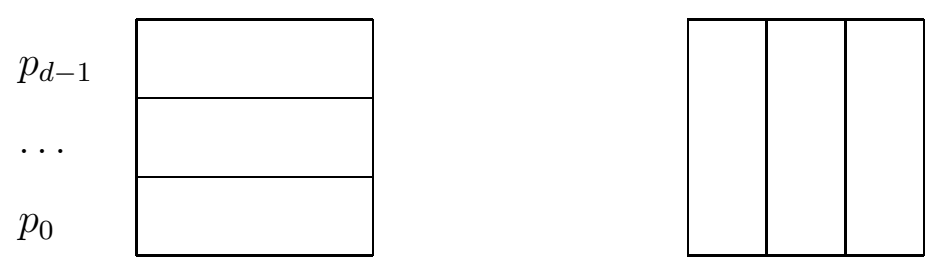

$$
q_{0} \quad \ldots \quad q_{d-1}
$$

Lemma 2.3. Let $P=I \times \omega$ be a multitile and assume its horizontal tiles are $p_{0}, \ldots, p_{d-1}$ and its vertical tiles are $q_{0}, \ldots, q_{d-1}$. If

$$
G_{p_{j}}=\left(\begin{array}{ll}
a_{j} & b_{j} \\
\overline{b_{j}} & \overline{a_{j}}
\end{array}\right)
$$


for $j=0, \ldots, d-1$, then

$$
G_{q_{m}}=\prod_{j=d-1}^{0}\left(\begin{array}{cc}
a_{j} & \gamma^{m j} b_{j} \\
\gamma^{m j} b_{j} & \overline{a_{j}}
\end{array}\right) .
$$

Here the product is to be read in descending order

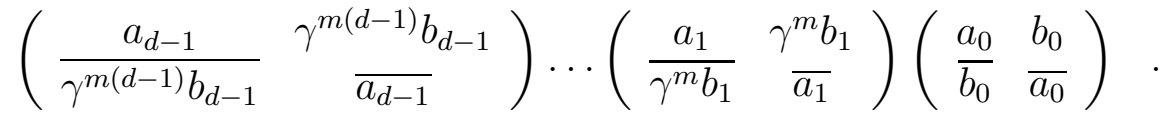

Proof: Let $k$ denote the left endpoint of $I$ and let $d^{\kappa}$ be the length of $I$. Let $p_{j}=I \times \omega_{j}$. By Lemma 2.1 it suffices to prove for $0 \leq m \leq d-1$ :

$$
G_{\omega_{j}}\left(k+d^{\kappa-1} m, \infty\right)=\left(\begin{array}{cc}
\frac{a_{j}}{\gamma^{m j} b_{j}} & \gamma^{m j} b_{j} \\
a_{j}
\end{array}\right)
$$

However, we have for $x \in \omega_{j}$ :

$$
\begin{gathered}
w\left(k+d^{\kappa-1} m, x\right)=\left(\gamma^{\sum_{\nu<\kappa-1} k_{\nu} x_{-1-\nu}}\right) \gamma^{m j}\left(\gamma^{\sum_{\nu \geq \kappa} k_{\nu} x_{-1-\nu}}\right) \\
=\gamma^{m j} w(k, x)
\end{gathered}
$$

Now (13) follows by the considerations in the proof of Lemma 2.2.

In the next section we will obtain a function $\beta: S U(1,1) \rightarrow \mathbb{R}_{0}^{+}$such that

$$
\frac{1}{C} \log |a| \leq \beta(G) \leq C \log |a|
$$

for some constant $C$ depending polynomially on $d$ and, with the notation of Lemma 2.3 ,

$$
\sum_{m=0}^{d-1} \beta\left(G_{p_{m}}\right) \leq d \sum_{j=0}^{d-1} \beta\left(G_{q_{j}}\right) .
$$

We will refer to $\beta$ as the swapping function and (14) as the swapping property. Assume for now this swapping function has been constructed. The rest of this section is to prove (8) using this function.

Let $K$ be a large integer and consider the rectangle $R=\left[0, d^{K}\right) \times\left[0, d^{K}\right)$. Let $\mathbf{p}_{\kappa}$ denote the set of all tiles $I \times \omega \subset R$ with $|I|=d^{\kappa}$. One can partition the tiles in $\mathbf{p}_{\kappa}$ into $d$-tuples such that each $d$-tuple consists of the horizontal tiles of a multitile. Applying (14) on each tuple we obtain

$$
\sum_{p \in \mathbf{p}_{\kappa}} \beta\left(G_{p}\right) \leq d \sum_{p \in \mathbf{p}_{\kappa+1}} \beta\left(G_{p}\right)
$$

By iterating this we obtain

$$
d^{-K} \sum_{p \in \mathbf{p}_{-K}} \beta\left(G_{p}\right) \leq d^{K} \sum_{p \in \mathbf{p}_{K}} \beta\left(G_{p}\right)
$$

Hence

$$
d^{-K} \sum_{p \in \mathbf{p}_{-K}} \log \left|a_{p}\right| \leq C d^{K} \sum_{p \in \mathbf{p}_{K}} \log \left|a_{p}\right|
$$

where $a_{p}$ denotes the upper left entry of $G_{p}$. Observe that since we have no control over $K$ it is very important that there are no further constants on the right hand side 
of (2.3) other than the constant $d$ which is the natural scaling constant (as we will see momentarily).

We may assume that the support of $F$ is contained in $\left[0, d^{K}\right)$. Then for a tile $p=$ $I \times\left[0, d^{K}\right)$ we have that $a_{p}$ is equal to $a(k, \infty)$ where $k$ is the left endpoint of $I$, or, by Lemma 2.2 where $k$ is any point in $I$. Thus the left hand side of (15) is equal to

$$
\int_{0}^{d^{K}} \log |a(k, \infty)| d k
$$

Thus it remains to show that the right hand side of (15) is less than

$$
C\|F\|_{2}^{2}
$$

for arbitrarily large $K$ and constant $C$ independent of $K$. Observe that (11) implies

$$
\frac{\partial}{\partial x}\left\|G_{\omega}\right\|_{o p} \leq\left\|W_{\omega}\right\|_{o p}\left\|G_{\omega}\right\|_{o p}
$$

which implies together with the intitial condition for $G$ at 0 :

$$
\left\|G_{\omega}(k, \infty)\right\|_{o p} \leq \exp \left(\int_{0}^{\infty}\left\|W_{\omega}\right\|_{o p}\right)
$$

This is Gronwall's inequality and - as has been mentioned before - implies the $L^{1}$ estimates claimed in Theorem 1.1. Continuing the present considerations we obtain

$$
\log \left\|G_{\omega}(k, \infty)\right\|_{o p} \leq\|F\|_{L^{1}(\omega)}
$$

We claim that the operator norm of a matrix of the type (10) is equal to $|a|+|b|$. This is clear in the case that $a$ and $b$ are real, in which it is easy to calculate the eigenvalues of the symmetric matrix. The general case can be obtained by multiplying the matrix from both sides by unitary diagonal matrices to reduce to the real case.

By Hölder's inequality we thus have

$$
\log \left(\left|a_{\omega}\right|+\left|b_{\omega}\right|\right) \leq\|F\|_{2}|\omega|^{1 / 2}
$$

By choosing $\omega$ small enough ( $K$ large enough), the right hand side can be made small. Thus we can assume $|a|$ is close to 1 and $b$ is close to 0 . Then we obtain

$$
\log \left(\left|a_{\omega}\right|+\left|b_{\omega}\right|\right) \geq \frac{1}{2}\left|b_{\omega}\right| \geq \frac{1}{4} \sqrt{\log \left|a_{\omega}\right|} .
$$

Hence

$$
d^{K} \sum_{p \in \mathbf{p}_{K}} \log \left|a_{p}\right| \leq 4 \sum_{p \in \mathbf{p}_{K}}\left\|F_{\omega}\right\|_{2}^{2}=4\|F\|_{2}^{2} .
$$

This gives the desired bound on the right hand side of (15) and completes the proof of inequality (8) in the case of compactly supported $F$.

If $F$ is not compactly supported then we will show later that the limit (7) exists almost everywhere. Assuming this for now, then (8) follows by Fatou's lemma. 


\section{THE SWAPPING FUNCTION}

In this section we will find for each $d>1$ a function

$$
\beta: S U(1,1) \rightarrow \mathbb{R}_{0}^{+}
$$

such that $\beta(G)$ is comparable to $\log |a|$ and we have the inequality (14). We call this inequality a swapping inequality and $\beta$ a swapping function because (14) swaps the vertical tiles to the horizontal tiles in a given multitile.

The case $d=2$ is particularly easy and we will do it first. The function $\beta$ simply can be chosen to be the logarithm of the Hilbert Schmidt norm of $G$. Here we define the Hilbert Schmidt norm of a matrix $G$ of the form (10) to be

$$
\|G\|_{H S}=\sqrt{|a|^{2}+|b|^{2}} \text {. }
$$

Observe that for $a$ near 1 we have

$$
\log \|G\|_{H S} \sim \log \left(|a|^{2}+|b|^{2}\right) \sim|b|^{2} \sim \log |a|
$$

and for large $a$ we have

$$
\log \|G\|_{H S} \sim \log \left(2|a|^{2}\right) \sim \log |a| .
$$

Thus $\beta(G)$ is comparable to $\log |a|$. We write

$$
A^{-*}:=\left(A^{-1}\right)^{*}
$$

The following lemma then says that the swapping inequality is true.

Lemma 3.1. If $A, B \in S U(1,1)$ then

$$
\log \|A B\|_{H S}+\log \left\|A B^{-*}\right\|_{H S} \leq 2 \log \|A\|_{H S}+2 \log \|B\|_{H S}
$$

Write

$$
A=\left(\begin{array}{cc}
a & b \\
\bar{b} & \bar{a}
\end{array}\right)
$$

and

$$
B=\left(\begin{array}{cc}
c & d \\
\bar{d} & \bar{c}
\end{array}\right), \quad B^{-1}=\left(\begin{array}{cc}
\bar{c} & -d \\
-\bar{d} & c
\end{array}\right), \quad B^{-*}=\left(\begin{array}{cc}
c & -d \\
-\bar{d} & \bar{c}
\end{array}\right)
$$

Then we have

$$
A B=\left(\begin{array}{cc}
a c+b \bar{d} & b \bar{c}+a \bar{d} \\
\bar{b} c+\bar{a} d & \overline{a c}+\bar{b} d
\end{array}\right), \quad A B^{-*}=\left(\begin{array}{cc}
a c-b \bar{d} & b \bar{c}-a \bar{d} \\
\bar{b} c-\bar{a} d & \overline{a c}-\bar{b} d
\end{array}\right)
$$

This gives

$$
\|A B\|_{H S}^{2}+\left\|A B^{-*}\right\|_{H S}^{2}=2\left(|a c|^{2}+|b d|^{2}+|b c|^{2}+|a d|^{2}\right)=2\|A\|_{H S}^{2}\|B\|_{H S}^{2} .
$$

Using the arithmetic mean-geometric mean inequality we obtain

$$
\|A B\|_{H S}\left\|A B^{-*}\right\|_{H S} \leq\|A\|_{H S}^{2}\|B\|_{H S}^{2}
$$

Taking logarithms proves the lemma.

We remark that the function $\beta(G):=\log |a|$ does not satisfy the swapping inequality in general. This can be seen from choosing $a, b, c$ positive and $d$ purely imaginary in the above example. 
Now consider $d \geq 3$. In this case one has to choose a more complicated swapping function. Indeed, in an appendix to this section we will sketch an argument that the logarithm of the Hilbert Schmidt norm does not satisfy the required swapping inequality.

Choose an $\epsilon$ sufficiently small. For the purpose of keeping track of polynomial growth in the parameter $d$ we remark that the choice $10^{-3} d^{-1}$ will be sufficient.

Let $r$ be the smallest positive number such that

$$
r^{2}-r^{3}=\epsilon^{10}+\epsilon^{20} \operatorname{arcsinh}(r) .
$$

Then $r$ is of the order $\epsilon^{5}$. We consider the following swapping function defined on $\mathbb{C}$

$$
\beta(z):=|z|^{2}-|z|^{3}
$$

if $|z| \leq r$ and

$$
\beta(z):=\epsilon^{10}+\epsilon^{20} \operatorname{arcsinh}(|z|)
$$

if $|z| \geq r$.

Finding this function was inspired by the discussion of Bellman functions in [12], whence the letter $\beta$ for this function. For a matrix $G$ of type (10) we will let $\beta(G)=\beta(b)$. By a discussion as in (16) and (17) it is clear that $\beta(G)$ is comparable to $\log |a|$ with constants growing polynomially in $\epsilon^{-1}$ and thus growing polynomially in $d$.

Clearly there is not a unique way to choose $\beta$. Our choice reflects in a very explicit way the two different types of behaviour for small and for large $|z|$ which will be apparent from the discussion below. Moreover, for each of the two regions our choice shows explicitly the leading order term $\left(|z|^{2}\right.$ and $\left.C^{-1} \operatorname{arcsinh}(|z|)\right)$ and a smaller order correction term which is used to estimate the nonlinear effects. The third order correction term for small $z$ could be replaced by any other power $|z|^{p}$ with $2<p<4$.

Given $d$ pairs $\left(a_{i}, b_{i}\right)$ of complex numbers with $\left|a_{i}\right|^{2}=1+\left|b_{i}\right|^{2}$ and a $d$-th root of unity $\gamma$ (in this section $\gamma$ shall not be a fixed primitive $d$-th root of unity but an arbitrary $d$-th root of unity) we define

$$
\left(\begin{array}{cc}
\frac{A_{\gamma}}{B_{\gamma}} & \frac{B_{\gamma}}{A_{\gamma}}
\end{array}\right)=\prod_{i=1}^{d}\left(\begin{array}{cc}
\frac{a_{i}}{\gamma^{i} b_{i}} & \gamma^{i} b_{i} \\
a_{i}
\end{array}\right) .
$$

The factors in this product do not commute, hence we emphasize that the product is understood in ascending order:

$$
\left(\begin{array}{cc}
\frac{a_{1}}{\gamma^{1} b_{1}} & \gamma^{1} b_{1} \\
\overline{a_{1}}
\end{array}\right)\left(\begin{array}{cc}
a_{2} & \gamma^{2} b_{2} \\
\overline{\gamma^{2} b_{2}} & \overline{a_{2}}
\end{array}\right) \ldots\left(\begin{array}{cc}
a_{d} & \gamma^{d} b_{d} \\
\gamma^{d} b_{d} & \overline{a_{d}}
\end{array}\right) .
$$

Also observe that in the last factor we have $\gamma^{d}=1$.

Lemma 3.2. Under the above hypotheses, we have

$$
\sum_{\gamma} \beta\left(B_{\gamma}\right) \leq d \sum_{i=1}^{d} \beta\left(b_{i}\right)
$$

where the sum on the left hand side runs over all d-th roots of unity.

This lemma clearly implies the desired swapping inequality (14).

Proof: We shall first consider the case when $\left|b_{i}\right| \leq r$ for all $i$. 
Observe that we can write $B_{\gamma}$ as a polynomial in the (for this matter viewed as independent) variables $a_{i}, b_{i}, \overline{a_{i}}$ and $\overline{b_{i}}$ for $i=1, \ldots, d$.

We claim that this polynomial is odd in the vector

$$
\mathbf{b}=\left(b_{1}, \overline{b_{1}}, \ldots, b_{d}, \overline{b_{d}}\right)
$$

This follows from the observation that the operation $G \rightarrow G^{-*}$ (see (18) and the lines thereafter) commutes with matrix products. Thus replacing the vector $\mathbf{b}$ by its negative replaces $B_{\gamma}$ by its negative. Thus $B_{\gamma}$ has to be an odd polynomial in $\mathbf{b}$.

Writing down the matrix product explicitly, we observe that the polynomial $B_{\gamma}$ is a sum of monomials of degree $d$, where each such monomial has exactly one entry from each of the matrices $G_{1}, \ldots, G_{d}$ as factor. Any choice of one entry from each matrix can appear in a monomial, provided the following row- and column conditions are satisfied: an entry from the $j$-th row of $G_{i}$ can appear only if an entry from the $j$-th column of $G_{i-1}$ appears, the entry form $G_{d}$ has to be from the second column, and the entry from $G_{1}$ has to be from the first row.

This together with oddness in $\mathbf{b}$ gives the crude estimate

$$
\left|B_{\gamma}\right| \leq \sum_{i}\left|b_{i}\right| \prod_{j \neq i}\left(\left|a_{i}\right|+\left|b_{i}\right|\right) \leq d r(1+2 r)^{d-1} \leq 2 d r
$$

The right hand side is of the order $\epsilon^{4}$ and thus we are well in the range such that we have an estimate

$$
\beta\left(B_{\gamma}\right) \leq\left|B_{\gamma}\right|^{2}-\left|B_{\gamma}\right|^{3}
$$

Studying now the polynomial of $B_{\gamma}$ more carefully, we extract those terms which are linear in $\mathbf{b}$. They are easily seen to be

$$
\sum_{i=1}^{d} \gamma^{i}\left(\prod_{j<i} a_{j}\right) b_{i}\left(\prod_{j>i} \overline{a_{j}}\right) .
$$

Moreover, since there are no terms quadratic in $\mathbf{b}$, we obtain the estimate

$$
\begin{gathered}
\left|B_{\gamma}-\sum_{i=1}^{d} \gamma^{i}\left(\prod_{j<i} a_{j}\right) b_{i}\left(\prod_{j>i} \overline{a_{j}}\right)\right| \\
\leq \sum_{i<j<k}\left|b_{i}\right|\left|b_{j}\right|\left|b_{k}\right| \prod_{l \neq i, j, k}\left|a_{l}\right|+\left|b_{l}\right| \leq 2 d^{3}\left|b_{m}\right|\left|b_{m^{\prime}}\right|^{2} .
\end{gathered}
$$

Here $m$ denotes the index such that $\left|b_{m}\right|$ is maximal among all $\left|b_{i}\right|$ and $m^{\prime}$ denotes the index such that $\left|b_{m^{\prime}}\right|$ is maximal among all $b_{i}$ with $i \neq m$.

Now we consider the polynomial for $\left|B_{\gamma}\right|^{2}=B_{\gamma} \overline{B_{\gamma}}$ and sum over all $\gamma$. Observe that upon the summation in $\gamma$, all terms of the polynomial of $\left|B_{\gamma}\right|^{2}$ which have a non-trivial power of $\gamma$ in the coefficient get canceled.

We are again interested in the lowest order terms in $\mathbf{b}$, which are the quadratic terms. Such terms appear when the $i$-th summand of (21) is multiplied by the complex conjugate of the $j$-th summand in (21). The power of $\gamma$ in such a term is trivial only if $i=j$. Thus 
the (in b) quadratic terms of $\sum_{\gamma}\left|B_{\gamma}\right|^{2}$ are precisely

$$
d \sum_{i=1}^{d}\left|b_{i}\right|^{2}\left(\prod_{j \neq i}\left|a_{j}\right|^{2}\right)
$$

Moreover, from the previous discussion we can easily see the estimate

$$
\left.\left|\sum_{\gamma}\right| B_{\gamma}\right|^{2}-\left.d \sum_{i=1}^{d}\left|b_{i}\right|^{2}\left(\prod_{j \neq i}\left|a_{j}\right|^{2}\right)\left|\leq 4 d^{4} r\right| b_{m}|| b_{m^{\prime}}\right|^{2} .
$$

Now we use the fact that $\left|a_{i}\right|^{2}=1+\left|b_{i}\right|^{2}$ to obtain

$$
\left.\left|\sum_{\gamma}\right| B_{\gamma}\right|^{2}-\left.d \sum_{i=1}^{d}\left|b_{i}\right|^{2}\left|\leq 6 d^{4} r\right| b_{m}|| b_{m^{\prime}}\right|^{2} \text {. }
$$

Next, we observe

$$
\frac{1}{d} \sum_{\gamma}\left|B_{\gamma}\right|^{3} \geq\left(\frac{1}{d} \sum_{\gamma}\left|B_{\gamma}\right|^{2}\right)^{3 / 2} \geq\left(\sum_{i=1}^{3}\left|b_{i}\right|^{2}\right)^{3 / 2}-\frac{3}{2} 6 d^{3} r\left|b_{m}\right|\left|b_{m^{\prime}}\right|^{2} .
$$

The last estimate followed from (24) and a trivial estimate on the slope of the function $x \rightarrow x^{3 / 2}$ in the interval $[0,1]$. Now the right hand side of the last display is equal to

$$
\begin{gathered}
\left|b_{m}\right|^{3}\left(1+\sum_{i \neq m}\left(\left|b_{i}\right| /\left|b_{m}\right|\right)^{2}\right)^{3 / 2}-9 d^{3} r\left|b_{m}\right|\left|b_{m^{\prime}}\right|^{2} \\
\geq\left|b_{m}\right|^{3}\left(1+\frac{3}{2} \sum_{i \neq m}\left(\left|b_{i}\right| /\left|b_{m}\right|\right)^{2}\right)-9 d^{3} r\left|b_{m}\right|\left|b_{m^{\prime}}\right|^{2} \\
\geq \sum_{i}\left|b_{i}\right|^{3}+\frac{1}{2}\left|b_{m}\right|\left|b_{m^{\prime}}\right|^{2}-9 d^{3} r\left|b_{m}\right|\left|b_{m^{\prime}}\right|^{2} \geq \sum_{i}\left|b_{i}\right|^{3}+\frac{1}{4}\left|b_{m}\right|\left|b_{m^{\prime}}\right|^{2} .
\end{gathered}
$$

Together with (24) we obtain

$$
\sum_{\gamma}\left|B_{\gamma}\right|^{2}-\left|B_{\gamma}\right|^{3} \leq d \sum_{i}\left|b_{i}\right|^{2}-\left|b_{i}\right|^{3}
$$

Together with (20) this proves the Lemma 3.2 in the present case $\left|b_{i}\right| \leq r$ for all $i$.

Now we consider the case at the other extreme that for at least two indices $i$ we have $\left|b_{i}\right| \geq r$. Denote by $I$ the set of indices $i$ for which $\left|b_{i}\right| \geq r$.

We observe that the quantity $\operatorname{arcsinh}|b|$ has the meaning of the logarithm of the operator norm of the matrix

$$
\left(\begin{array}{ll}
a & b \\
\bar{b} & \bar{a}
\end{array}\right)
$$

By elementary calculus using $|a|^{2}=1+|b|^{2}$ this is equivalent to the statement that the operator norm of this matrix is $|a|+|b|$. This however has been observed in Section 2 .

In particular we obtain for each $\gamma$ :

$$
\operatorname{arcsinh}\left|B_{\gamma}\right| \leq \sum_{i} \operatorname{arcsinh}\left|b_{i}\right| \quad .
$$


Since by elementary calculus we always have

$$
\beta\left(B_{\gamma}\right) \leq \epsilon^{10}+\epsilon^{20} \operatorname{arcsinh}\left|B_{\gamma}\right|
$$

we obtain

$$
\begin{gathered}
\beta\left(B_{\gamma}\right) \leq \epsilon^{10}+\epsilon^{20} \sum_{i} \operatorname{arcsinh}\left|b_{i}\right| \\
\leq \epsilon^{10}(1-|I|)+\sum_{i \in I} \beta\left(b_{i}\right)+\epsilon^{20} \sum_{i \notin I} \operatorname{arcsinh}\left|b_{i}\right| \\
\leq-\epsilon^{10}+\sum_{i \in I} \beta\left(b_{i}\right)+\epsilon^{20} d r \leq \sum_{i \in I} \beta\left(b_{i}\right) .
\end{gathered}
$$

Summing over $\gamma$ proves Lemma 3.2 in this case.

The same reasoning as in the previous case can be applied if there is only one index $i$ such that $\left|b_{i}\right| \geq r$ but there is at least one other index $j$ such that $\left|b_{j}\right|>\epsilon r$. The latter implies that

$$
\epsilon^{20} \operatorname{arcsinh}\left|b_{j}\right| \leq \beta\left(b_{j}\right)-\epsilon^{15}
$$

Namely, the left hand side is less than $\epsilon^{20}$, while $\beta\left(b_{j}\right)$ is at least $\epsilon^{14}$. Thus we have

$$
\begin{gathered}
\beta\left(B_{\gamma}\right) \leq \beta\left(b_{i}\right)+\beta\left(b_{j}\right)-\epsilon^{15}+\epsilon^{20} \sum_{k \neq i, j} \operatorname{arcsinh}\left|b_{k}\right| \\
\leq \beta\left(b_{i}\right)+\beta\left(b_{j}\right) .
\end{gathered}
$$

This proves the Lemma 3.2 in the given case.

It remains to prove the case when there is one index $i$ such that $\left|b_{i}\right| \geq r$ and for all other indices $j \neq i$ we have $\left|b_{j}\right| \leq \epsilon r$. We extract from (22):

$$
\gamma^{-i} B_{\gamma}=b_{i}+\sum_{j>i} \gamma^{j-i} a_{i} b_{j}+\sum_{j<i} \gamma^{j-i} \overline{a_{i}} b_{j}+E
$$

with

$$
|E| \leq 4 d^{2}\left(\left|a_{i}\right|+\left|b_{i}\right|\right)\left|b_{m^{\prime}}\right|^{2} \leq 8 d^{2}\left(1+\left|b_{i}\right|\right)\left|b_{m^{\prime}}\right|^{2}
$$

Here $m^{\prime}$ is the index such that $\left|b_{m^{\prime}}\right|$ is maximal among all $\left|b_{j}\right|$ with $j \neq i$. Observe that under the given assumptions the term $b_{i}$ is large compared to the linear terms in $b_{j}, j \neq i$, which in turn are large compared to $E$. Indeed, we observe the estimate

$$
\left|\sum_{j<i} \gamma^{j-i} a_{i} b_{j}+\sum_{j>i} \gamma^{j-i} \overline{a_{i}} b_{j}+E\right| \leq 2 d\left(1+\left|b_{i}\right|\right)\left|b_{m^{\prime}}\right| .
$$

Our goal is to make a Taylor expansion of the function $f: z \rightarrow \operatorname{arcsinh}|z|$ near the point $b_{i}$. Let $\lambda$ denote the linear form which is the derivative of $f$ at $b_{i}$ and let $\rho$ denote the quadratic form which is the second derivative of $f$ at an appropriate point within distance $2 d\left(1+\left|b_{i}\right|\right)\left|b_{m^{\prime}}\right|$ of $b_{i}$.

Then we obtain from Taylor's theorem

$$
\begin{gathered}
\sum_{\gamma} \operatorname{arcsinh}\left|B_{\gamma}\right|=\sum_{\gamma} \operatorname{arcsinh}\left|\gamma^{-i} B_{\gamma}\right| \\
=d \operatorname{arcsinh}\left|b_{i}\right|+\sum_{\gamma} \lambda\left(\sum_{j<i} \gamma^{j-i} a_{i} b_{j}+\sum_{j>i} \gamma^{j-i} \overline{a_{i}} b_{j}+E\right)+F
\end{gathered}
$$


with

$$
|F| \leq \sum_{\gamma}|\rho|\left|\sum_{j<i} \gamma^{j-i} a_{i} b_{j}+\sum_{j>i} \gamma^{j-i} \overline{a_{i}} b_{j}+E\right|^{2}
$$

Using that

$$
\sum_{\gamma} \gamma^{j-i}=0
$$

for all $j \neq i$ we obtain

$$
\sum_{\gamma} \operatorname{arcsinh}\left|B_{\gamma}\right|=d \operatorname{arcsinh}\left|b_{i}\right|+\sum_{\gamma} \lambda(E)+F
$$

From elementary calculus we obtain

$$
\begin{gathered}
|\lambda| \leq\left(1+\left|b_{i}\right|^{2}\right)^{-1 / 2} \\
|\rho| \leq 20\left|b_{i}\right|^{-1}\left(1+\left|b_{i}\right|^{2}\right)^{-1 / 2} .
\end{gathered}
$$

Hence

$$
\sum_{\gamma} \operatorname{arcsinh}\left|B_{\gamma}\right| \leq d \operatorname{arcsinh}\left|b_{i}\right|+50 r^{-1} d^{3}\left|b_{m}\right|^{2}
$$

and

$$
\sum_{\gamma} \beta\left(B_{\gamma}\right) \leq d \beta\left(b_{i}\right)+\epsilon^{20} 50 r^{-1} d^{3}\left|b_{m}\right|^{2} \leq d \sum_{j} \beta\left(b_{j}\right) .
$$

This proves the Lemma 3.2 in the last case and thus completes the proof.

We close this section by showing that for $d=3$ we cannot choose $\beta(G)$ to be log $\|G\|_{H S}$ if we want the swapping inequality (14) to hold. The rest of this section is irrelevant for the purpose of proving Theorem 1.4.

Let $\gamma$ denote a third root of unity. Define

$$
\left(\begin{array}{ll}
a & b \\
c & d
\end{array}\right)_{\gamma}=\left(\begin{array}{cc}
a & \gamma b \\
\gamma^{2} c & d
\end{array}\right)
$$

We aim to find matrices $A, B, C$ in $S U(1,1)$ such that

$$
\prod_{\gamma}\left\|A_{\gamma \gamma} B_{\gamma} C\right\|_{2}>\|A\|^{3}\|B\|^{3}\|C\|^{3}
$$

where the product on the left hand side goes over all three third roots of unity. We shall present such matrices $A, B, C$ with real entries. Thus we write

$$
A=\left(\begin{array}{ll}
a & b \\
b & a
\end{array}\right), \quad B=\left(\begin{array}{ll}
c & d \\
d & c
\end{array}\right), \quad C=\left(\begin{array}{cc}
e & f \\
f & e
\end{array}\right) .
$$

By homogeneity of (25) the requirement that $A, B, C$ are in $S U(1,1)$ can be relaxed to the requirement that they have nonzero determinant and $|a|>|b|,|c|>|d|$, and $|e|>|f|$. Indeed we will produce an example satisfying the latter constraints and

$$
a^{2}+b^{2}=1, \quad c^{2}+d^{2}=1, \quad e^{2}+f^{2}=1 .
$$

In particular the right hand side of (25) is equal to 1. 
The matrix $A_{\gamma \gamma} B_{\gamma} C$ is equal to

$$
A=\left(\begin{array}{cc}
a c e+\gamma a d f+\gamma b d e+\gamma^{2} b c f & * \\
\gamma b c e+\gamma^{2} b d f+\gamma^{2} a d e+a c f & *
\end{array}\right)
$$

where the unspecified entries in the second column are the same as the diagonally opposite terms.

We calculate the Hilbert Schmidt norm squared of this matrix, which is the sum of modulus sqared of the two indicated entries.. Observe that squaring the entries and multiplying out gives pure squares (the modulus square of a summand) and mixed terms (product of two different summands with proper complex conjugation). The pure squares simply add up to

$$
\left(a^{2}+b^{2}\right)\left(c^{2}+d^{2}\right)\left(e^{2}+f^{2}\right)=1 .
$$

To calculate the mixed terms it helps to observe that we may divide the second entry by $\gamma$, then the two entries are alike but with $a$ and $b$ interchanged. Using $a^{2}+b^{2}=1$ and $\bar{\gamma}=\gamma^{2}$ we obtain for the mixed terms

$$
\begin{aligned}
& +c d e f \gamma^{2}+2 a b c d e^{2} \gamma^{2}+2 a b c^{2} e f \gamma+2 a b d^{2} e f+2 a b c d f^{2} \gamma^{2}+c d e f \gamma^{2} \\
& +c d e f \gamma+2 a b c d e^{2} \gamma+2 a b c^{2} e f \gamma^{2}+2 a b d^{2} e f+2 a b c d f^{2} \gamma+c d e f \gamma .
\end{aligned}
$$

Now we set $\alpha=a b$ and $\beta=e f$. Thus it will suffice to produce $\alpha, \beta \in[0,1 / 2]$. Using $e^{2}+f^{2}=1$ we obtain for the square of the Hilbert Schmidt norm

$$
\left(1+4 \alpha \beta d^{2}\right)+\left(2 \alpha \beta c^{2}+2 \alpha c d+2 \beta c d\right) \gamma+\left(2 \alpha \beta c^{2}+2 \alpha c d+2 \beta c d\right) \gamma^{2} .
$$

Now we observe for any three numbers $K, L, M$ the formula

$$
\prod_{\gamma} K+L \gamma+M \gamma^{2}=K^{3}+L^{3}+M^{3}-3 K L M .
$$

Namely, expanding the left hand side, clearly the coefficients in front of $K^{3}, K^{2} L$, and $K^{2} M$ are 1, 0, 0 ; the latter two because the sum of the third roots of unity is 0 . By multiplying each factor on the left hand side by $\gamma\left(\gamma^{2}\right)$ we see that the left hand side is invariant under cyclic permutations of $K, L, M$. Thus it remains to check that the factor 3 in front of $K L M$ is correct. This however follows from letting $K=L=M=1$.

Now fix $\beta>0$ and choose $\alpha$ and $d$ very small but nonzero such that

$$
2 \alpha \beta c^{2}+2 \beta c d=0 \text {. }
$$

Let $K=1+2 \alpha \beta d^{2}+2 \alpha \beta d^{2}, L=2 \alpha c d, M=2 \alpha c d$. Then $L^{3}, M^{3}$, and $3 K L M$ are small of order at least $\alpha^{2} d^{2}$. However,

$$
K^{3}=1+12 \alpha \beta d^{2}+O\left(\alpha^{2} d^{2}\right) .
$$

Thus the left hand side of (25) can be made bigger than 1 .

\section{Proof of inequality (9)}

This section is very close to the known existing proofs of Carleson's theorem in the classical linear case. We follow closely [15]. For example Corollary 4.2 corresponds to a Bessel inequality in the linear case. In the current non-linear setting it is convenient to estimate the contribution of a single tree pointwise outside an exceptional set (in the spirit of the original proof by Carleson [5]) instead of using any $L^{p}$ estimate, because of 
the ease of pointwise summing a geometrically decaying sequence using a quasi triangle inequality, see the calculation beginning with (37).

We shall first assume that $F$ is compactly supported.

We are interested in the dependence of constants on $d$. It will help to introduce a constant $\Gamma$ which (other than the constant $C$ ) does not change from line to line and has polynomial growth in $d$. The constants $C$ in this section will be independent of $d$.

If $G$ is a matrix in $S U(1,1)$ and $a$ is its first entry, we shall write

$$
|G|:=|a| \text {. }
$$

Choose $\Gamma$ so that

$$
\Gamma^{-1} \log |G| \leq \beta(G) \leq \Gamma \log |G|
$$

By construction of $\beta$ this constant grows polynomially in $d$.

Orthogonality of disjoint tiles. We define a partial ordering on tiles by $p<p^{\prime}$ if $I \subset I^{\prime}$ and $\omega^{\prime} \subset \omega$. Recall that all intervals $I$ and $\omega$ are $d$-adic and assumed to be half open (containing the left but not the right endpoint). Therefore two such intervals are either disjoint or one is contained in the other. Since tiles have area one we conclude that two tiles are comparable if and only if they have non-empty intersection.

We observe that we have the following corollary of Lemma 3.2.

Corollary 4.1. Let $\mathbf{q}$ be a finite set of pairwise disjoint tiles and let $\mathbf{p}$ be a finite set of tiles such that for all $q \in \mathbf{q}$ we have

$$
q \subset \bigcup_{p \in \mathbf{p}} p
$$

and for all $q \in \mathbf{q}$ and $p \in \mathbf{p}$ we have $q<p$ whenever $p$ and $q$ have nonempty intersection. Then

$$
\sum_{q \in \mathbf{q}}\left|I_{q}\right| \beta\left(G_{q}\right) \leq \sum_{p \in \mathbf{p}}\left|I_{p}\right| \beta\left(G_{p}\right)
$$

Proof:

If $\mathbf{p}$ has none or one element, then (26) is trivial because $\mathbf{q}$ has to be a subset of $\mathbf{p}$. Fix $\mathbf{p}$, by induction we may assume the corollary has been proved for all subsets of $\mathbf{p}$. Now choose q. By cancelling equal summands on both sides of (26) and using the result for subsets of $\mathbf{p}$ we may assume that $\mathbf{q}$ and $\mathbf{p}$ are disjoint. We may assume $\mathbf{q}$ is nonempty and choose $q$ such that $l=\left|I_{q}\right|$ is minimal. Since the possible values of $l$ are discrete and bounded above by $\sum_{P \in \mathbf{p}}\left|I_{p}\right|$, we may assume by induction that the statement of the corollary is true for all values of $l$ larger than a given $l_{0}$, and we have to prove the statement under the assumption $l=l_{0}$. Now we use induction on the number $n$ of tiles $q$ in $\mathbf{q}$ which satisfy $\left|I_{q}\right|=l$. Again by induction we may fix an $n_{0}$ and assume that the statement is true for all $n<n_{0}$ and we have to prove the statement assuming $n=n_{0}$.

Now pick a tile $q \in \mathbf{q}$ such that $\left|I_{q}\right|=l$. It is the vertical tile of a multitile $Q$. We claim

1. $Q \subset \bigcup_{p \in \mathbf{p}} p$

2. Any vertical tile in $Q$ is either an element of $\mathbf{q}$ or it is disjoint from all tiles in $\mathbf{q}$. Assuming these two claims for now, we observe that it suffices to prove the statement of the corollary for $\mathbf{q}^{\prime}$ which is the union of $\mathbf{q}$ and the set of vertical tiles in $Q$. Observe that $l^{\prime}=l$ and $n^{\prime}<n+d-1$ where $l^{\prime}$ and $n^{\prime}$ are defined analogously to $l$ and $n$. By the 
swapping inequality (14) it suffices to prove the statement for $\mathbf{q}^{\prime \prime}$ which is equal to $\mathbf{q}^{\prime}$ with all vertical tiles of $Q$ removed and all horizontal tiles of $Q$ added in. Observe that $l^{\prime \prime} \geq l$ and, if $l=l^{\prime \prime}$, then $n^{\prime \prime}<n$. Thus the statement of the corollary follows by induction.

It remains to prove the above two claims. To see the first claim, pick $(k, x) \in Q$. There is a $\left(k_{0}, x\right) \in q$. Then there is a $p \in \mathbf{p}$ with $\left(k_{0}, x\right) \in p$. Since $p \cap q \neq \emptyset$ we have by assumptions on $\mathbf{p}$ that $q<p$. Thus $I_{p}$ is a $d$-adic interval, strictly containing $I_{q}$ because of $p \neq q$. By $d$-adicity $I_{Q} \subset I_{p}$ and hence $(k, x) \in p$ which had to be proved.

To see the second claim pick a vertical tile $q^{\prime}$ of $Q$ and assume that $q^{\prime} \cap q^{\prime \prime} \neq \emptyset$ for some $q^{\prime \prime} \in \mathbf{q}$. By minimality of the choice of $I_{q}$ we have $I_{q^{\prime}} \subset I_{q^{\prime \prime}}$. If this inclusion was strict, then $q^{\prime \prime} \cap q \neq \emptyset$ which is impossible. Hence $I_{q}^{\prime}=I_{q^{\prime \prime}}$ and hence $q=q^{\prime}$. This proves the second claim and completes the proof of the corollary.

Corollary 4.2. Let $q$ be a set of pairwise disjoint tiles. Then

$$
\sum_{q \in \mathbf{q}}\left|I_{q}\right| \beta\left(G_{q}\right) \leq C \Gamma \int|F(x)|^{2} d x .
$$

Proof: This follows from the previous corollary by a limiting argument as in the proof of (8).

Selecting trees. We define an ordering on multitiles analogous to the ordering on tiles. Thus $P<P^{\prime}$ for two multitiles $P=I \times \omega$ and $P^{\prime}=I^{\prime} \times \omega^{\prime}$ if $I \subset I^{\prime}$ and $\omega^{\prime} \subset \omega$.

A set $\mathbf{P}$ of multitiles is called convex, if for any three multitiles $P<P^{\prime}<P^{\prime \prime}$ with $P, P^{\prime \prime} \in \mathbf{P}$ we can conclude $P^{\prime} \in \mathbf{P}$.

An ordered splitting of a set $\mathbf{P}$ of multitiles is a decomposition of $\mathbf{P}$ into a disjoint union

$$
\mathbf{P}=\bigcup_{n \in N} \mathbf{P}_{n}
$$

where $N$ is a subset of the integers (and possibly $\infty$ ) and $P \in \mathbf{P}_{n}, P^{\prime} \in \mathbf{P}_{n^{\prime}}$ with $P<P^{\prime}$ imply $n \leq n^{\prime}$. Observe that if $\mathbf{P}$ is convex, then the components $\mathbf{P}_{n}$ of an ordered splitting are again convex.

A tree is a set $T$ of multitiles which has a maximal element with respect to the ordering of multitiles. This maximal element is called the top of the tree and denoted by $P_{T}$.

Each element $P$ of a tree other than the top itself has a distinguished index $j_{P} \in$ $0, \ldots, d-1$ attached to it such that $p_{j_{P}}$ is the unique horizontal subtile of $P$ which intersects the tree top. For the top $P_{T}$ of a tree we define $j_{P_{T}}=d-1$. Observe that we have suppressed the dependence of $j_{P}$ on the given tree in the notation.

We define the size of a collection of multitiles by

$$
\operatorname{size}(\mathbf{P})=\sup _{T \subset \mathbf{P}}\left|I_{T}\right|^{-1} \sum_{P \in T} \sum_{j<j_{P}}\left|I_{P}\right| \beta\left(G_{p_{j}}\right)
$$

where the sup is taken over all trees in $\mathbf{P}$. For a given tree $T$ the tiles $p_{j}$ occuring in the sum on the right hand side of (27) are pairwise disjoint.

The above Corollary 4.2 implies the following lemma: 
Lemma 4.3. Let $\mathbf{P}$ be a convex set of multitiles. Then we can decompose $\mathbf{P}$ into an ordered splitting $\mathbf{P}_{1} \cup \mathbf{P}_{2}$ such that

$$
\operatorname{size}\left(\mathbf{P}_{2}\right)<2^{-4} \operatorname{size}(\mathbf{P})
$$

and $\mathbf{P}_{1}$ is the (not necessarily disjoint) union of a collection $\mathbf{T}$ of trees such that

$$
\sum_{T \in \mathbf{T}}\left|I_{T}\right| \leq C \Gamma \operatorname{size}(\mathbf{P})^{-1}\|F\|_{2}^{2}
$$

and each tree $T \in \mathbf{T}$ with top $P_{T}$ has the saturation property that if $P<P_{T}$ for some $P \in \mathbf{P}_{1}$ then $P \in T$.

Proof: Set $\alpha=2^{-4} \operatorname{size}(\mathbf{P})$.

We select recursively for $n=1,2,3 \ldots$ a tree $T_{n}$. Suppose we have already chosen $T_{m}$ for all $m<n$. If there is a tree $\tilde{T}_{n}$ in

$$
\mathbf{P}^{n}:=\mathbf{P} \backslash \bigcup_{m<n} T_{m}
$$

with size larger than $\alpha$, then we choose one such tree with top $P_{n}$ say such that the upper endpoint of $\omega_{P_{n}}$ is minimal. The tree $T_{n}$ is then the maximal tree in $\mathbf{P}$ with respect to set inclusion with top $P_{n}$.

We iterate this tree selection until we reach an $n=N$ such that there is no tree in $\mathbf{P}^{n}$ with size larger than $\alpha$. If this is the case, we stop the selection and define $\mathbf{P}_{1}$ to be the union of trees selected. Define $\mathbf{P}_{2}=\mathbf{P} \backslash \mathbf{P}_{1}$. By maximality of each selected tree it is clear that the splitting of $\mathbf{P}$ into $\mathbf{P}_{1}$ and $\mathbf{P}_{2}$ is ordered and that each selected tree satisfies the saturation property of the lemma. Moreover, by the stopping condition for the tree selection it is clear that $\mathbf{P}_{2}$ satisfies the size estimate (28).

It remains to prove the bound (29). By Corollary 4.1 it suffices to show that the set of tiles $p_{j}$ with $P \in \tilde{T}_{n}$ for some $1 \leq n \leq N$ and $j<j_{P}$ is a set of pairwise disjoint tiles. Suppose to get a contradiction that $p_{j}<p^{\prime}{ }_{j^{\prime}}$ for two distinct such tiles. Then $P$ belongs to a tree $\tilde{T}_{n}$ and $P^{\prime}$ belongs to a tree $\tilde{T}_{n^{\prime}}$. By $d$-adicity it is easy to see that the upper endpoint of $\omega_{P_{n}}$ is greater than the upper endpoint of $\omega_{P_{n^{\prime}}}$, in particular $n \neq n^{\prime}$ and $n<n^{\prime}$. But the geometry of $p_{n^{\prime}}$ qualifies it to be in the tree $T_{n}$, which is a contradiction to the maximality of $T_{n}$.

This proves Lemma 4.3 .

By iterating this lemma we obtain:

Corollary 4.4. If $\mathbf{P}$ is any finite set of tiles, we can decompose it into an ordered splitting

$$
\mathbf{P}=\mathbf{P}_{\infty} \cup \bigcup_{\mathbf{k} \in \mathbf{Z}} \mathbf{P}_{\mathbf{k}}
$$

such that

$$
\operatorname{size}\left(\mathbf{P}_{\mathbf{k}}\right) \leq 2^{-4 \mathbf{k}}
$$

and $\mathbf{P}_{\mathbf{k}}$ is the union of a collection $\mathbf{T}_{\mathbf{k}}$ of trees such that

$$
\sum_{T \in \mathbf{T}_{\mathbf{k}}}\left|I_{T}\right| \leq C \Gamma 2^{4 \mathbf{k}}\|F\|_{2}^{2}
$$


and each tree $T \in \mathbf{T}_{\mathbf{k}}$ with top $P$ contains all elements $P^{\prime} \in \mathbf{P}_{\mathbf{k}}$ with $P^{\prime}<P$. Moreover, $\operatorname{size}\left(P_{\infty}\right)=0$. If the set $\mathbf{P}$ is convex, then all trees in $\mathbf{T}_{\mathbf{k}}$ are convex.

A John - Nirenberg type estimate for a single tree. Given a convex tree $T$, we shall be concerned with the following function defined on $I_{T}$

$$
M_{T}(k)=\sup _{k \in I, I^{\prime}: I \subset I^{\prime} \subsetneq I_{T}} \log \left|\prod_{P \in T: I \subset I_{P} \subset I^{\prime}} \prod_{j<j_{P}} G_{p_{j}}(k)\right| .
$$

Here the product is to be understood in the natural order of descending size of $I_{P}$ and descending $j$ : If $\left|I_{P}\right|<\left|I_{P^{\prime}}\right|$, and $j=j_{P}>0, j^{\prime}=j_{P^{\prime}}>0$, then the corresponding factors appear in the order

$$
\ldots G_{p_{j^{\prime}-1}} G_{p_{j^{\prime}-2}^{\prime}} \ldots G_{p_{0}^{\prime}} \ldots G_{p_{j-1}} G_{p_{j-2}} \ldots G_{p_{0}} \ldots .
$$

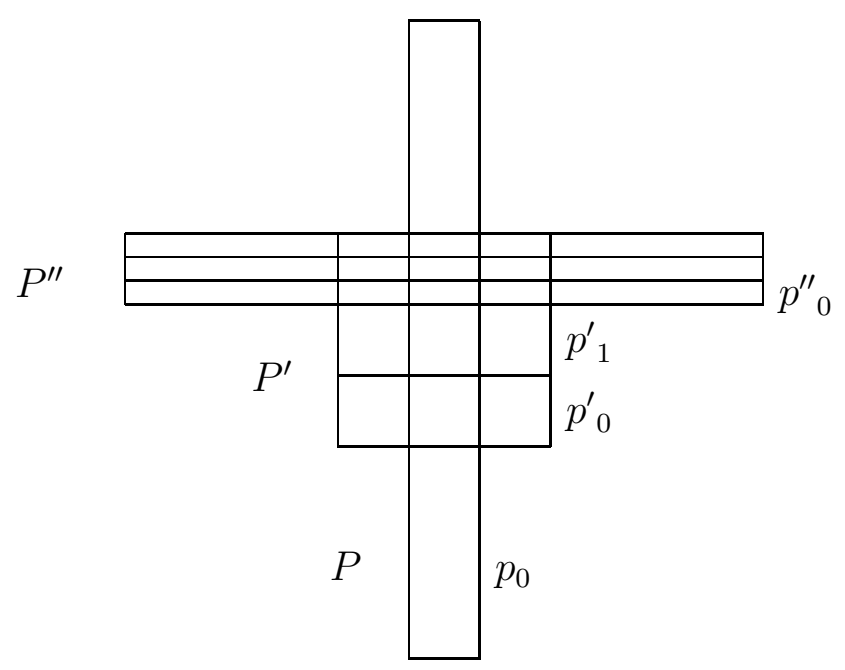

We have the John-Nirenberg type lemma:

Lemma 4.5. Let $T$ be a convex tree. Then for every integer $\mu \geq 0$ we have

$$
\left|\left\{k \in I_{T}: M(k)>4 d \Gamma 2^{2 \mu} \operatorname{size}(T)\right\}\right| \leq 2^{-c \mu^{2}}\left|I_{T}\right|
$$

for some small universal constant $c$.

Remark: This inequality gives less decay in $\mu$ on the right hand side than the usual John- Nirenberg inequality. The loss is due to our approach to dealing with the quasi triangle inequality in (31) instead of a triangle inequality.

Proof:

Observe that it suffices to prove the Lemma for large $\mu$.

Let $P_{T}$ be the top of the tree and $\xi$ be the lower endpoint of the interval $\omega_{P_{T}}$. Observe that all intervals $\omega_{p_{j}}$ for $p_{j}$ appearing in the product in (30) lie below $\xi$. Therefore we do not change the value of $M_{T}$ if we restrict $F$ to $[0, \xi]$. Therefore we shall assume for the purpose of proving this lemma that $F$ is supported in $[0, \xi]$.

It suffices to prove a similar estimate for the simpler variant 


$$
\tilde{M}_{T}(k)=\sup _{I: k \in I} \log \left|\prod_{P \in T: I \subset I_{P} \subsetneq I_{T}} \prod_{j<j_{P}} G_{p_{j}}(k)\right| .
$$

This follows from writing

$$
\prod_{P \in T: I \subset I_{P} \subset I^{\prime}} \prod_{j<j_{P}} G_{p_{j}}(k)=\left(\prod_{P \in T: I^{\prime} \subsetneq I_{P} \subsetneq I_{T}} \prod_{j<j_{P}} G_{p_{j}}(k)\right)^{-1}\left(\prod_{P \in T: I \subset I_{P} \subsetneq I_{T}} \prod_{j<j_{P}} G_{p_{j}}(k)\right)
$$

and estimating both factors on the right hand side by $\tilde{M}_{T}$. Namely, observe that for all $G, G^{\prime} \in S U(1,1)$ we have

$$
|G|=\left|G^{-1}\right|
$$

and the quasi triangle inequality

$$
\log \left|G G^{\prime}\right| \leq 2 \log |G|+2 \log \left|G^{\prime}\right|
$$

The latter follows from Lemma 3.1. Thus we obtain the pointwise estimate

$$
M_{T}(k) \leq 4 \tilde{M}_{T}(k),
$$

which reduces the matter to estimating $\tilde{M}_{T}$.

We first prove for $\lambda \geq 1$ the following estimate:

$$
\left|\left\{k: \tilde{M}_{T}(k)>\Gamma \lambda \operatorname{size}(T)\right\}\right| \leq \lambda^{-1}\left|I_{T}\right| .
$$

This follows from the estimate

$$
\left|\left\{k: \tilde{\tilde{M}}_{T}(k)>\lambda \operatorname{size}(T)\right\}\right| \leq \lambda^{-1}\left|I_{T}\right| .
$$

for the modified function

$$
\tilde{\tilde{M}}_{T}(k)=\sup _{I: k \in I} \beta\left(\prod_{P \in T: I \subset I_{P}, P \neq P_{T}} \prod_{j<j_{P}} G_{p_{j}}(k)\right)
$$

because $\beta(G)$ and $\log |a|$ are comparable by a factor of $\Gamma$.

Let $P$ be a multitile of the tree $T$, let $q$ be a vertical tile in $P$, and assume $k \in I_{q}$. Then we have by support assumption on $F$, convexity of the tree, and Lemma 2.1:

$$
G_{q}(k)=\prod_{P^{\prime} \in T: I_{P} \subset I_{P^{\prime}}, P^{\prime} \neq P_{T}} \prod_{j<j_{P}^{\prime}} G_{p_{j}}(k) .
$$

Namely, it is an elementary geometric observation that the intervals $\omega_{p_{j}}$ on the right form a partition of the interval $\omega_{q} \cap(-\infty, \xi)$.

Let $\mathbf{q}$ be the set of maximal tiles in the set of all tiles $q$ which are vertical tile of some $P \in T$ and which satisfy

$$
\beta\left(G_{q}\right) \geq \lambda \operatorname{size}(T)
$$

Observe that the set estimated on the left hand side of (33) is contained in the union of $I_{q}$ with $q \in \mathbf{q}$. Furthermore observe that the union of all $q \in \mathbf{q}$ is covered by the union of all $P \in \mathbf{T}$. Hence it is covered by the top multitile $P_{T}$ and all horizontal tiles $p_{j}$ of multitiles $P \in T$ and $j \neq j_{P}$. 
An application of Lemma 3.1 gives

$$
\sum_{q \in \mathbf{q}}\left|I_{q}\right| \beta\left(G_{q}\right) \leq \sum_{P \in \mathbf{T}, P \neq P_{T}} \sum_{j<j_{P}}\left|I_{P}\right| \beta\left(G_{p_{j}}\right)
$$

Here we have used that on the right hand side we do not have to include the terms with $P=P_{T}$ or $j>j_{P}$ because they give zero contribution thanks to support assumption on $F$.

This implies

$$
\sum_{q \in \mathbf{q}}\left|I_{q}\right| \leq \lambda^{-1}\left|I_{T}\right|
$$

which proves (33) and therefore also (32).

Now we bootstrap (32) to the desired estimate for $\tilde{M}_{T}$. This step is analoguous to the bootstrapping argument that can be used to prove the usual John- Nirenberg inequality, which is why we say Lemma 4.5 is of John-Nirenberg type. It suffices to prove:

$$
\left|\left\{k: \tilde{M}_{T}(k)>2^{\mu+2} d \Gamma \operatorname{size}(T)\right\}\right| \leq 2^{-\mu}\left|\left\{k: \tilde{M}_{T}(k)>2^{\mu} d \Gamma \operatorname{size}(T)\right\}\right| .
$$

Namely, given this estimate, we have by iteration for every integer $\mu \geq 0$

$$
\left|\left\{k: \tilde{M}_{T}(k)>2^{2 \mu} d \Gamma \operatorname{size}(T)\right\}\right| \leq 2^{-c \mu^{2}}\left|\left\{k: \tilde{M}_{T}(k)>d \Gamma \operatorname{size}(T)\right\}\right| \leq 2^{-c \mu^{2}}\left|I_{T}\right| .
$$

This will prove the desired estimate. We prove (34).

Let $\mathbf{P}$ be the set of maximal multitiles in $P \in T$ such that

$$
\max \left(\log \left|G_{q_{1}}\right|, \ldots, \log \left|G_{q_{d}}\right|\right) \geq 2^{\mu} d \Gamma \operatorname{size}(T)
$$

where $q_{1}, \ldots, q_{d}$ are the vertical subtiles of $P$. For each such $P$ let $P^{\prime} \in T$ be the minimal multitile in $T$ which is larger than $P$. (The proof trivializes if there is no such multitile because then $P=P_{T}$.) Assume that $I_{P}$ is equal to $q^{\prime}{ }_{j}$.

Then, by maximality,

$$
\log \left|G_{q_{j}^{\prime}}\right| \leq 2^{\mu} d \Gamma \operatorname{size}(T)
$$

and moreover, for every $k \in I_{P}$,

$$
\sup _{I: I_{P} \subset I} \log \left|\prod_{P^{\prime} \in T: I \subset I_{P^{\prime}}, I_{P^{\prime}} \neq I_{T}} \prod_{j<j_{P^{\prime}}} G_{p_{j}^{\prime}}(k)\right| \leq 2^{\mu} d \Gamma \operatorname{size}(T) .
$$

Observing that every subtree of $T$ has size at most size $(T)$ and applying (32) to the subtree of all $P^{\prime \prime} \in T$ with $I_{P^{\prime \prime}} \subset I_{P}$ and using the quasi triangle inequality (31) we obtain:

$$
\left|\left\{k \in I_{P}: \tilde{M}_{T}(k)>2\left(2^{\mu}+2^{\mu}\right) d \Gamma \operatorname{size}(T)\right\}\right| \leq 2^{-\mu} d^{-1}\left|I_{P}\right| \quad .
$$

Since the intersection of $\left|I_{P}\right|$ and the set where $\tilde{M}_{k}>d^{\mu} \operatorname{size}(T)$ has measure at least $d^{-1}\left|I_{P}\right|$ (this argument is one of two places in the proof of (9) where we lose a power of $d$ in the dependence on $d$ other than the loss due to $\Gamma$ ) we obtain:

$$
\left|\left\{k \in I_{T}: \tilde{M}_{T}(k)>2^{\mu+2} d \Gamma \operatorname{size}(T)\right\}\right| \leq 2^{-\mu}\left|\left\{k \in I_{T}: \tilde{M}_{k}>2^{\mu} d \Gamma \operatorname{size}(T)\right\}\right| .
$$

This proves (34) and completes the proof of Lemma 4.5. 
The Carleson theorem. By restricting the set of tiles to those inside a large square $\left[0, d^{K}\right) \times\left[0, d^{K}\right)$ and a subsequent limiting argument as in Section 2 we may assume the set $\mathbf{P}$ of all multitiles is finite.

We are aiming to show that there exists a $C$ such that for each $\lambda>0$ we have

$$
\left|\left\{k: \sup _{x} \log |G(k, x)|>C d \lambda^{-1}\right\}\right| \leq \Gamma^{2}\|F\|_{2}^{2} \lambda \text {. }
$$

Fix $\lambda$.

Decompose $\mathbf{P}$ into $\mathbf{P}_{\mathbf{k}}$ according to Corollary 4.4, where

$$
\Gamma \operatorname{size}\left(\mathbf{P}_{\mathbf{k}}\right) \leq 2^{-4 \mathbf{k}}
$$

and $\mathbf{P}_{\mathbf{k}}$ is the union of a collection $\mathbf{T}_{\mathbf{k}}$ of trees such that

$$
\sum_{T \in \mathbf{T}_{\mathbf{k}}}\left|I_{T}\right| \leq C \Gamma^{2} 2^{4 \mathbf{k}}\|F\|_{2}^{2}
$$

We define an exceptional set $E=\bigcup E_{\mathbf{k}}$.

Let $K$ be a negative integer of large modulus to be determined later. For $\mathbf{k}<K$ we define

$$
E_{\mathbf{k}}=\bigcup_{T \in \mathbf{T}_{\mathbf{k}}} I_{T}
$$

and obtain

$$
\left|E_{\mathbf{k}}\right| \leq C \Gamma^{2} 2^{4 \mathbf{k}}\|F\|_{2}^{2}
$$

For $\mathbf{k}>K$ we define

$$
E_{\mathbf{k}}=\bigcup_{T \in \mathbf{T}_{\mathbf{k}}}\left\{k \in I_{T}: M_{T}(k) \geq 4 d 2^{2(\mathbf{k}-K)} 2^{-4 \mathbf{k}}\right\} .
$$

By the John-Nirenberg type Lemma we have for $T \in \mathbf{T}_{\mathbf{k}}$

$$
\left|\left\{k \in I_{T}: M_{T}(k) \geq 4 d 2^{2(\mathbf{k}-K)} 2^{-4 \mathbf{k}}\right\}\right| \leq 2^{-c(\mathbf{k}-K)^{2}}\left|I_{T}\right| .
$$

Thus

$$
\left|E_{\mathbf{k}}\right| \leq C \Gamma^{2} 2^{-c(\mathbf{k}-K)^{2}} 2^{4 \mathbf{k}}\|F\|_{2}^{2}=C \Gamma^{2} 2^{-c(\mathbf{k}-K)^{2}} 2^{4(\mathbf{k}-K)} 2^{4 K}\|F\|_{2}^{2} .
$$

If we choose $K$ maximal with $\lambda>C 2^{4 K}$ for a certain $C$, then

$$
|E| \leq \sum_{\mathbf{k} \in \mathbf{Z}}\left|E_{\mathbf{k}}\right| \leq \Gamma^{2} \lambda\|F\|_{2}^{2}
$$

It remains to prove that for $k \notin E$ and every $x$ we have

$$
\log |G(k, x)| \leq C d \lambda^{-1}
$$

for some constant $C$. Fix $x$. We can write

$$
G(k, x)=\prod_{P \in T} \prod_{j<j_{P}} G_{\omega_{p_{j}}}(k, x)
$$

where $T$ is the convex tree of all multitiles $P$ such that $k \in I_{P}$ and $x \in \omega_{P}$ and $j_{P}$ for $P \in T$ is the unique index such that $x \in p_{j_{P}}$.

Let $T_{\mathbf{k}}$ be the intersection of $T$ with $\mathbf{P}_{k}$. Since the sets $\mathbf{P}_{\mathbf{k}}$ form an ordered splitting, the sets $T_{\mathbf{k}}$ are convex trees. Moreover, each $T_{\mathbf{k}}$ is contained in a tree $\tilde{T}_{\mathbf{k}}$ of $\mathbf{T}_{\mathbf{k}}$ by the saturation property of the trees in $\tilde{T}_{\mathbf{k}}$. 
Denote the top of $T_{\mathbf{k}}$ by $P_{\mathbf{k}}$. Observe that if $P \in T_{\mathbf{k}}$ and $P \neq P_{\mathbf{k}}$, then the number $j_{P}$ defined with respect to $T$ is the same as the one defined with respect to $T_{\mathbf{k}}$.

Hence we can estimate the contribution of the tree $T_{\mathbf{k}}$ using Lemma 4.5 and the quasi triangle inequality as follows

$$
\begin{gathered}
\log \left|\prod_{P \in T_{\mathbf{k}}} \prod_{j<j_{P}} G_{\omega_{p_{j}}}(k, x)\right| \\
\leq 2 \log \left|\prod_{j<j_{P_{\mathbf{k}}}} G_{\omega_{p_{j}}}(k, x)\right|+2 \log \left|\prod_{P \in T_{\mathbf{k}}, P \neq P_{\mathbf{k}}} \prod_{j<j_{P_{\mathbf{k}}}} G_{\omega_{p_{j}}}(k, x)\right| \\
\leq 2 \log \left|\prod_{j<j_{P_{\mathbf{k}}}} G_{\omega_{p_{j}}}(k, x)\right|+2 M_{\tilde{T}_{\mathbf{k}}}(k) .
\end{gathered}
$$

Here $p_{j}$ in the first summand of (36) and the preceding line is a horizontal tile of $P_{\mathbf{k}}$. To estimate the first term we use that $\log |G|$ is comparable to $\beta(G)$ and the the swapping inequality to obtain

$$
\begin{gathered}
\quad \log \left|\prod_{j<j_{P_{\mathbf{k}}}} G_{\omega_{p_{j}}}(k, x)\right| \\
\leq \Gamma \beta\left(\prod_{j<j_{P_{\mathbf{k}}}} G_{\omega_{p_{j}}}(k, x)\right) \\
\leq d \Gamma \sum_{j<j P_{\mathbf{k}}} \beta\left(G_{\omega_{p_{j}}}\right) \leq d 2^{-4 \mathbf{k}}
\end{gathered}
$$

The last inequality follows by observing that $\left\{P_{\mathbf{k}}\right\}$ constitutes a tree by itself which is controlled in size because $P \in \mathbf{P}_{\mathbf{k}}$. Observe that in this argument we lose a factor $d$.

Thus, by choice of $x$.

$$
\log \left|\prod_{P \in T_{\mathbf{k}}} \prod_{j<j_{P}} G_{\omega_{p_{j}}}(k, x)\right| \leq C d \Gamma 2^{2(\mathbf{k}-K)} 2^{-4 \mathbf{k}} .
$$

The trees $T_{\mathbf{k}}$ with $\mathbf{k}<K$ are empty, because $x$ is not in the exceptional set. Moreover, by finiteness assumption on the set $\mathbf{P}$ there is a $K^{\prime}$ so that $T_{\mathbf{k}}$ is empty for $\mathbf{k}>K^{\prime}$ and $\mathbf{k} \neq \infty$. (The constant $C$ is not allowed to depend on $K^{\prime}$ )

Then we have

$$
G(k, x)=\left(\prod_{P \in T_{\infty}} \prod_{j<j_{P}} G_{\omega_{p_{j}}}(k, x)\right)\left(\prod_{K \leq \mathbf{k} \leq K^{\prime}} \prod_{P \in T_{\mathbf{k}}} \prod_{j<j_{P}} G_{\omega_{p_{j}}}(k, x)\right)
$$

where as usual the product has to be read in the correct order. The factor coming from $T_{\infty}$ can be discarded since it gives a unitary matrix.

By the quasi triangle inequality we have

$$
\log |G(k, x)| \leq 4 C d 2^{-4 K} .
$$


Namely, we can prove inductively

$$
\begin{gathered}
\log \left|\prod_{K^{\prime \prime} \leq \mathbf{k} \leq K^{\prime}} \prod_{P \in T_{\mathbf{k}}} \prod_{j<j_{P}} G_{\omega_{p_{j}}}(k, x)\right| \\
\leq 2 \log \left|\prod_{P \in T_{K^{\prime \prime}}} \prod_{j<j_{P}} G_{\omega_{p_{j}}}(k, x)\right|+2 \log \left|\prod_{K^{\prime \prime}+1 \leq \mathbf{k} \leq K^{\prime}} \prod_{P \in T_{\mathbf{k}}} \prod_{j<j_{P}} G_{\omega_{p_{j}}}(k, x)\right| \\
\leq 2 C d 2^{2\left(K^{\prime \prime}-K\right)} 2^{-4 K^{\prime \prime}}+2 C d 2^{2\left(K^{\prime \prime}-K\right)} 2^{-4 K^{\prime \prime}} \\
\leq C d 2^{\left.2\left(\left(K^{\prime \prime}-1\right)-K\right)\right)} 2^{-4\left(K^{\prime \prime}-1\right)}
\end{gathered}
$$

where contrary to our standing convention the constant $C$ for induction purpose is the same in all appearances in this calculation.

This proves inequality (35) and therefore completes the proof of inequality (9) in the case of compactly supported $F$.

It remains to discuss the case of not necessarily compactly supported $F \in L^{2}(\mathbb{R})$. We first prove that the limit (7) exists almost everywhere. It suffices to fix small $\epsilon$ and prove that the limit exists for all $k$ outside a set of measure $\epsilon$. This can be done by decomposing the positive real axis into intervals $\left[0, x_{1}\right),\left[x_{1}, x_{2}\right)$, etc, such that the $L^{2}$ norms of the restrictions of $F$ to the intervals $\omega_{j}=\left[x_{j}, x_{j+1}\right)$ decay very rapidly in $j$. This implies by the Plancherel inequality (8) that for $k$ outside a small set (of size $\epsilon / 2$ ) the values $\log \left|G_{\omega_{j}}\right|$ are still very rapidly decaying, so that one can use the triangle inequality

$$
\log \left|G_{1} G_{2}\right| \leq \log \left|G_{1}\right|+\log \left\|G_{2}\right\|_{o p}
$$

to show that the sequence $\log \left|G_{\left[0, x_{j}\right)}\right|$ is a Cauchy sequence. Using (9) for for the restriction of $F$ to each interval $\left[x_{j}, x_{j+1}\right)$ one can observe that the operator norms of the matrices $G_{\left[x_{j}, x\right)}$ with $x_{j}<x<x_{j+1}$ are small for large $j$ and all $k$ outside a set of measure $\epsilon / 2$. Using the triangle inequality (38) one can show that for $k$ outside a set of measure $\epsilon$ the limit of $\log \left|G_{[0, x)}\right|$ exists. This proves existence of the limits in (7). Similar arguments as these make it straight forward to prove (9) for arbitrary potentials $F \in L^{2}([0, \infty))$.

\section{Multilinear expansions}

Writing the differential equation (6) as an integral equation

$$
G(k, x)=\left(\begin{array}{ll}
1 & 0 \\
0 & 1
\end{array}\right)+\int_{-\infty}^{x}\left(\begin{array}{cc}
\frac{0}{F(x) w(k, t)} & F(x) w(k, t) \\
0
\end{array}\right) G(k, t) d t
$$

we can use Picard iteration to obtain the formal solution

$$
G(k, x)=\left(\begin{array}{ll}
1 & 0 \\
0 & 1
\end{array}\right)+\sum_{n=1}^{\infty} \int_{t_{1}<\cdots<t_{n}<x} \prod_{j=n}^{1}\left(\begin{array}{cc}
\frac{0}{F\left(t_{j}\right) w(k, t)} & F\left(t_{j}\right) w(k, t) \\
0
\end{array}\right) d t_{j} .
$$

Christ and Kiselev [6], [0] prove convergence for almost every $k$ of this formal expansion if $F \in L^{p}(\mathbb{R})$ with $p<2$, and they use the expansion to show the maximal Hausdorff Young inequality (they work on a different model of the nonlinear Fourier transform, but their arguments apply to this case too). In [11] it has been shown that the higher order terms of the Fourier analogue of this expansion are unbounded for $F \in L^{2}$ and therefore not very well suited to be used to prove a nonlinear Carleson theorem.

In this section we show that a similar discussion as in 11 applies in the $d$-adic setting provided $d \geq 3$. More precisely we will focus on the quadratic term in the above expansion 
and on the case $d=3$ and prove Proposition 5.1 below. The arguments generalize to $d>3$ and to the higher-linear terms, but we shall not elaborate on this because our main point can be made clear for this special case.

The quadratic term in the above expansion is a diagonal matrix with entries

$$
Q(F)(k, x)=\int_{t_{1}<t_{2}<x} F\left(t_{1}\right) w\left(k, t_{1}\right) \overline{F\left(t_{2}\right) w\left(k, t_{2}\right)} d t_{1} d t_{2}
$$

and the complex conjugate of $(40)$.

We consider

$$
M(F)(k)=\sup _{x}\left|\int_{t_{1}<t_{2}<x} F\left(t_{1}\right) w\left(k, t_{1}\right) \overline{F\left(t_{2}\right) w\left(k, t_{2}\right)} d t_{1} d t_{2}\right| .
$$

The following proposition implies that there is no reasonable a priori bound for the size of this function in terms of the $L^{2}$ norm of $F$.

Proposition 5.1. Let $d=3$. There is an $\epsilon>0$ such that for each $N>0$ there is a finite $d$-adic step function $F$ with $L^{2}$ norm 1 such that

$$
\|\{k: M(F)(k)>N\}\|>\epsilon \text {. }
$$

Proof:

Pick a large integer $N$. In the proof of Lemma 2.2 we have seen that for a given tile $p=I \times \omega$ we can write

$$
w(k, x) 1_{I}(k) 1_{\omega}(x)=w_{p}(k) \overline{\widehat{w}_{p}(x)}
$$

for some functions $w_{p}$ and $\widehat{w}_{p}$ which have constant modulus on $I$ and $\omega$ respectively and which we may assume to have $L^{2}$ norm 1 . We observe for $x \in \omega$ :

$$
\int w_{p}(k) \overline{w(k, x)} d k=\int w_{p}(k) \overline{w_{p}(k)} \widehat{w}_{p}(x) d k=\widehat{w}_{p}(x)
$$

Thus, relying on the well known fact that the Cantor group Fourier transform is an isometry in $L^{2}$ (this can be shown by a linearized version of the arguments in Section 2), we see that the integral on the left hand side of (42), which is the Walsh-Fourier transform of $w_{p}$, has to vanish outside $\omega_{P}$ and thus $\widehat{w_{p}}$ is indeed the Cantor group Fourier transform of $w_{p}$.

Consider the tiles

$$
p_{j}=I_{j} \times \omega_{j}:=\left[3^{-N} j, 3^{-N}(j+1)\right) \times\left[3^{N} j, 3^{N}(j+1)\right)
$$

for $j=0, \ldots, 3^{N}-1$ and set

$$
F(x):=\sum_{j=0}^{3^{N}-1} F_{j}(x):=\sum_{j=0}^{3^{N}-1} 3^{-N / 2} \widehat{w}_{p_{j}}(x) .
$$

Thus $\|F\|_{2}=1$.

Observe that the intervals $\omega_{j}$ above form a partition of the interval $\left[0,3^{2 N}\right)$. For $k \in$ $[0,1]$ let $x(k)$ be the left endpoint of the unique interval $\omega_{j}$ which contains $3^{2 N} k$. Consider for $k \in[0,1)$ :

$$
\tilde{Q}(F)(k)=\int_{t_{1}<t_{2}<x(k)} F\left(t_{1}\right) w\left(k, t_{1}\right) \overline{F\left(t_{2}\right) w\left(k, t_{2}\right)} d t_{1} d t_{2}
$$


We shall show that the imaginary part of $\tilde{Q}$ is large on a big set:

$$
\{k \in[0,1):|\Im(\tilde{Q}(F)(k))|>N / 4\}>1 / 3,
$$

which will prove the proposition. Our argument works only for the imaginary part. Indeed, the real part of $\tilde{Q}$ can be seen to satisfy good estimates. This is the reason why our argument works only for $d \geq 3$. If $d=2$, then the characters $w(k, x)$ are purely real and our argument does not work. We do not know whether for $d=2$ the series (39) converges for genuinly complex $F \in L^{2}$ in a reasonable sense.

We may split

$$
\tilde{Q}(F)(k)=\sum_{0 \leq j, j^{\prime} \leq 3^{N}-1} \int_{t_{1}<t_{2}<x(k)} F_{j}\left(t_{1}\right) w\left(k, t_{1}\right) \overline{F_{j^{\prime}}\left(t_{2}\right) w\left(k, t_{2}\right)} d t_{1} d t_{2}
$$

If $j>j^{\prime}$ then the integrand is zero on the domain of integration, thus we may disregard these terms. Likewise, if $3^{N} j^{\prime} \geq x(k)$, the integrand is zero. If $j<j^{\prime}<3^{-N} x(k)$, then the constraint $t_{1}<t_{2}<x(k)$ in the domain of integration is superfluous and we can write

$$
\begin{gathered}
\int_{t_{1}<t_{2}<x(k)} F_{j}\left(t_{1}\right) w\left(k, t_{1}\right) \overline{F_{j^{\prime}}\left(t_{2}\right) w\left(k, t_{2}\right)} d t_{1} d t_{2} \\
=\int F_{j}\left(t_{1}\right) w\left(k, t_{1}\right) d t_{1} \int \overline{F_{j^{\prime}}\left(t_{2}\right) w\left(k, t_{2}\right)} d t_{2} \\
=w_{p_{j}}(k) \overline{w_{p_{j^{\prime}}}(k)}=0 .
\end{gathered}
$$

Thus it only remains to consider the terms with $j=j^{\prime}<3^{-N} x(k)$ :

$$
\tilde{Q}(F)(k)=\sum_{0 \leq j<3^{-N} x(k)} \int_{t_{1}<t_{2}} F_{j}\left(t_{1}\right) w\left(k, t_{1}\right) \overline{F_{j}\left(t_{2}\right) w\left(k, t_{2}\right)} d t_{1} d t_{2}
$$

For each $t_{1}<t_{2}, t_{1}, t_{2} \in \omega_{j}$ there is a minimal 3-adic interval $\omega \subset \omega_{j}$ such that $t_{1}$ and $t_{2}$ are both contained in $\omega$. Then $t_{1}$ and $t_{2}$ are in different 3-adic subintervals $\omega_{(m)}$ and $\omega_{\left(m^{\prime}\right)}$ of the next smaller generation of $\omega$. Indeed, $m<m^{\prime}$. We split $\tilde{Q}(F)$ according to the size of $\omega$ as follows:

$$
\tilde{Q}(F)(k)=\sum_{\kappa \leq N} \sum_{0 \leq j<3^{-N} x(k)} \sum_{|\omega|=3^{\kappa}} \sum_{0 \leq m<m^{\prime} \leq 2} \int_{\omega_{(m)}} F_{j}\left(t_{2}\right) w\left(k, t_{2}\right) d t_{2} \int_{\omega_{\left(m^{\prime}\right)}} \overline{F_{j}}\left(t_{1}\right) \overline{w\left(k, t_{1}\right)} d t_{1}
$$

Now fix $\kappa$ and $j$. Let the $-\kappa$-th coefficient in the ternary expansion of $k$ be $k_{-\kappa}$ and the $-\kappa$-th coefficient in the ternary expansion of $3^{-N} j$ be $j_{N-\kappa}$. Then we observe that for $m=0,1,2$

$$
\int_{\omega_{(m)}} F_{j}\left(t_{2}\right) w\left(k, t_{2}\right) d t_{2}=\gamma^{m k_{\kappa}-m j_{N-\kappa}} \int_{\omega_{(0)}} F_{j}\left(t_{2}\right) w\left(k, t_{2}\right) d t_{2}
$$

since there is a bijection of $\omega_{(0)}$ to $\omega_{(m)}$ given by switching the $\kappa-1$-st coefficient of each element in $\omega_{(0)}$ from 0 to $m$. Moreover

$$
\left|\int_{\omega_{(m)}} F_{j}\left(t_{2}\right) w\left(k, t_{2}\right) d t_{2}\right|=3^{-N+\kappa-1}
$$


provided $\omega \subset\left[3^{N} j, 3^{N}(j+1)\right)$ and $3^{-N} j$ and $k$ are in the same 3 -adic interval $I_{(j)}$ of length $|\omega|^{-1}$. Otherwise the integral on the left hand side is zero. Thus

$$
\begin{gathered}
\Im \sum_{|\omega|=3^{\kappa}} \sum_{1 \leq m<m^{\prime} \leq 3} \int_{\omega_{(m)}} F_{j}\left(t_{2}\right) w\left(k, t_{2}\right) d t_{2} \int_{\omega_{\left(m^{\prime}\right)}} \overline{F_{j}}\left(t_{1}\right) \overline{w\left(k, t_{1}\right)} d t_{1} \\
=1_{I_{(j)}}(k) 3^{-N+\kappa-2} \Im \sum_{1 \leq m<m^{\prime} \leq 3} \gamma^{\left(m-m^{\prime}\right)\left(k_{\kappa}-j_{N-\kappa}\right)}
\end{gathered}
$$

The imaginary part of the sum

$$
\sum_{1 \leq m<m^{\prime} \leq 3} \gamma^{\left(m-m^{\prime}\right)\left(k_{\kappa}-j_{N-\kappa}\right)}
$$

is equal to 0 if $k_{\kappa}-j_{N-\kappa}=0$, it is equal to $-\Im(\gamma)$ if $k_{\kappa}-j_{N-\kappa}=1$ or -2 , and it is equal to $\Im(\gamma)$ if $k_{\kappa}-j_{N-\kappa}=-1$ or 2 .

Now we add the terms (43) over all $0<j<3^{-N} k$, i.e. we consider

$$
\Im \sum_{0 \leq j<3^{-N} x(k)} \sum_{|\omega|=3^{\kappa}} \sum_{0 \leq i<i^{\prime} \leq 2} \int_{\omega_{i}} F_{j}\left(t_{2}\right) w\left(k, t_{2}\right) d t_{2} \int_{\omega_{i^{\prime}}} \overline{F_{j}}\left(t_{1}\right) \overline{w\left(k, t_{1}\right)} d t_{1} .
$$

By the previous remarks it suffices to count the terms for which the contribution (43) is equal to $-\Im(\gamma)$ and the number of terms for which it is equal to $\Im(\gamma)$. If $k$ is in the left third of a 3 -adic interval of length $3^{-\kappa}$ then we do not have a nonzero contribution from any $j$ because the constraint $3^{-N} j<k$ and the constraint that $3^{-N} j$ and $k$ are in the same 3 -adic interval of length $3^{-\kappa}$ implies that $3^{-N} j$ is in the left third of the same 3 -adic interval of length $3^{-\kappa}$ as $k$ and thus $j_{N-\kappa}=0$. By the discussion of (44) we therefore see that (45) is equal to 0 .

If $k$ is in the middle third of a 3 -adic interval of length $3^{-\kappa}$, then we get a contribution if $3^{-N} j$ is in the left third of that interval. There are $3^{N-\kappa-1}$ such values of $j$, thus (45) is equal to $-3^{-3} \Im(\gamma)$.

If $k$ is in the right third of a 3-adic interval of length $3^{-\kappa}$, then we get as many $j$ in the left third as in the middle third, and their contributions cancel each other, thus (45) is equal to 0 .

Now summing (45) over all $\kappa \leq N$ reduces to counting the number of scales $\kappa$ for which $k$ is in the middle third of a 3 -adic interval of length $3^{-\kappa}$. We may restrict attention to $\kappa \geq 0$ because we assume $k \in[0,1)$ and hence $k$ is always in the left third of any 3 -adic interval of length $3^{-\kappa}$ if $\kappa<0$.

Thus

$$
\Im(\tilde{Q}(F))(k)=-3^{-3} \Im(\gamma) \#\left\{0 \leq \kappa \leq N: k_{-\kappa-1}=1\right\}
$$

Since for each scale exactly one third (in measure) of all numbers in $[0,1)$ are in the middle interval of a 3 -adic interval of that scale, we obtain

$$
\int_{0}^{1} \Im(\tilde{Q}(F))(k)=-(N+1) 3^{-4} \Im(\gamma)
$$

Moreover, clearly

$$
\sup _{k \in[0,1)}|\Im(\tilde{Q}(F))(k)|=(N+1) 3^{-3} \Im(\gamma)
$$


Thus

$$
\left\|\left\{k:|\Im(\tilde{Q}(F))(k)| \geq(N+1) 3^{-5} \Im(\gamma)\right\}\right\| \geq 3^{-2} .
$$

This proves 5.1 since the choice of $N$ was arbitrary.

\section{ApPEndix}

In this section we prove the formula (4). The proof uses complex contour integration, which is essentially the only method we know to prove the inequality. It is a global argument, which should be contrasted to the argument in Section 2 for the Cantor group case, which uses local methods. The local methods are useful in proving Carleson's theorem, while global methods are hard to adapt.

The argument in this section is well known, variants of it go back at least as far as the article by Buslaev and Faddeev [4] or the work by Verblunsky [16], [17] in the discrete case.

Let $F$ be a compactly supported, complex valued, smooth function on $\mathbb{R}$. Consider the solution to (2) with initial condition $G(-\infty)=\mathrm{id}$. Writing this initial value problem as an integral equation and using Picard iteration as in Section 5 gives the solution as a formal expansion

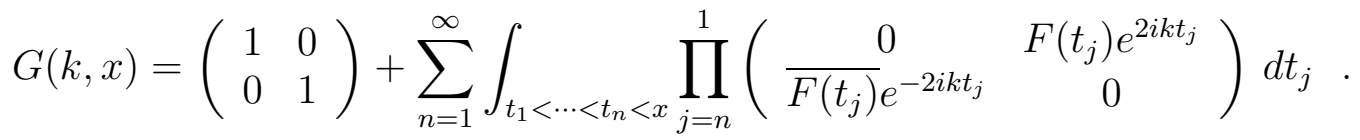

Indeed, this expansion is easily seen to converge using that the $L^{1}$ norm of $F$ is finite and a symmetry argument for the integration domain to obtain a factor $1 / n$ ! for the $n$-th multilinear term. At $x=\infty$ we obtain for the first entry $a(k)$ of $G(k, \infty)$

$$
a(k)=1+\sum_{n=1}^{\infty} \int_{t_{1}<t_{2}<\cdots<t_{2 n}} \prod_{j=1}^{n} F\left(t_{2 j-1}\right) \overline{F\left(t_{2 j}\right)} e^{2 i k\left(t_{2 j}-t_{2 j-1}\right)} d t_{2 j-1} d t_{2 j} .
$$

This function $a(k)$ extends holomorphically to $k$ in the half plane $\Im(k) \geq 0$ because it is a summable superposition of functions of the form $e^{i k t}$ with $t>0$. Assume for now that $a(k)$ does not have any zeros in the closed upper half plane, we will prove this at the end of this section.

Then we can define a function $\log (a(k))$ in the upper half plane. We choose the branch of the logarithm so that for $|k| \rightarrow \infty$ we have $\log (a(k)) \rightarrow 0$. It will become clear momentarily that this is well defined.

We consider the counter clockwise contour integral over a large semicircle $C=C_{1}+C_{2}$ where $C_{1}=[-r, r]$ and $C_{2}=\{k:|k|=r, \operatorname{Im}(k) \geq 0\}$. We show that on $C_{2}$ only the first nontrivial term in the expansion of $a(k)$ gives a contribution to the integral. We do a partial integration for this term

$$
\begin{gathered}
\int_{t_{1}<t_{2}} F\left(t_{2}\right) \overline{F\left(t_{1}\right)} e^{2 i k\left(t_{2}-t_{1}\right)} d t_{2} d t_{1} \\
=\int_{s>0} \int_{t} F(t+s) \overline{F(t)} e^{2 i k s} d t d s \\
=-\frac{1}{2 i k} \int_{t} F(t) \overline{F(t)} d t-\frac{1}{2 i k} \int_{s>0} \int_{t} F^{\prime}(t+s) \overline{F(t)} e^{2 i k s} d t d s
\end{gathered}
$$




$$
=-\frac{1}{2 i k}\|F\|_{2}^{2}+O\left(k^{-2}\right) \text {. }
$$

Here the estimate on the remainder term can be seen by one further partial integration.

For all the other terms in the expansion we can do partial integration in all variables $s_{j}=t_{2 j-1}-t_{2 j}$ so as to get the estimate $O\left(k^{-2}\right)$ or even better for all these terms. Thus we have for large $|k|$

$$
\log (a(k))=-\frac{1}{2 i k}\|F\|_{2}^{2}+O\left(k^{-2}\right) .
$$

Doing the integration on $C_{2}$ we obtain

$$
\int_{C_{2}} \log (a(k)) d k=-\frac{\pi}{2}\|F\|_{2}^{2}+O\left(r^{-1}\right) .
$$

Since the contour integral over $C$ vanishes, we obtain in the limit $r \rightarrow \infty$

$$
\int_{\mathbb{R}} \log (a(k)) d k=\frac{\pi}{2}\|F\|_{2}^{2},
$$

which implies (国) because the right hand side is real.

It remains to prove that $a$ does not have any zeros in the upper half plane. To this end write $a(k, x)$ and $b(k, x)$ for the entries in the first column of $G(k, x)$ and consider the quantity

$$
|a(k, x)|^{2}\left|e^{-2 i k x}\right|^{2}-|b(k, x)|^{2} .
$$

Writing $\Re(k)=\sigma$ and $\Im(\kappa)=\tau$, the partial derivative in the $x$ variable of this expression is

$$
\begin{aligned}
2 \Re\left[F(x) e^{2 i \sigma x} e^{2 \tau x} b(k, x) \bar{a}(k, x)\right. & \left.-\bar{F}(x) e^{-2 i \sigma x} e^{2 \tau x} a(k, x) \bar{b}(k, x)\right]+2 \tau|a(k, x)|^{2} e^{2 \tau x} \\
& =4 \tau|a(k, x)|^{2} e^{4 \tau x} .
\end{aligned}
$$

The latter is always positive for $\tau>0$. Since (46) is equal to

$$
\left|e^{-2 i k x}\right|^{2}
$$

for $x$ near $-\infty$, we conclude that (46) is positive for all $x$ and all $\tau>0$. This proves that $a(x, k)$ is nonzero for such $x$ and $\tau$. For $\tau=0$ we observe by a similar argument that $|a(k, x)|^{2}-\left.b(k, x)\right|^{2}$ is constant equal to 1 and thus $a$ has no zeros on the real axis neither.

\section{REFERENCES}

[1] Ablowitz, M.J., Kaup, D.J., Newell, A.C., Segur, H., The inverse scattering transform - Fourier analysis for nonlinear problems Studies in Applied Mathematics 53 [1974] pp. 249-315.

[2] Beals, R. and Coifman, R., Scattering and inverse scattering for first order systems on the line Comm. Pure Appl. Math. 37 [1984], no. 1, pp. 39-90.

[3] Billard, P. Sur la convergence presque partout de la séries Walsh-Fourier des fonctions de l'espace $L^{2}(0,1)$ Studia Math 28 [1966/67] pp. 363-388.

[4] Buslaev, V.S., and Faddeev, L. D., On formulas for traces of a Sturm-Liouville singular differential operator Dokl. Akad. Nauk SSSR, 132, No. 1, [1960] pp. 13-16.

[5] Carleson, L, On convergence and growth of partial sums of Fourier series Acta Math. 116 [1966], pp. 135-157.

[6] Christ, M., Kiselev, A., Maximal functions associated to filtrations, J. Funct. Anal. 179 [2001], no 2., pp. 409-425. 
[7] Christ, M., Kiselev, A., WKB asymptotic behaviour of almost all generalized eigenfunctions of onedimensional Schrödinger operators with slowly decaying potentials, J. Funct. Anal. 179 [2001], no 2., pp. 426-447.

[8] Deift, P. and Killip, R. On the absolutely continuous spectrum of one-dimensional Schrödinger operators with square summable potentials Commun. Math. Phys. 203 [1999] pp. 341-347.

[9] Hunt, R. On the convergence of Fourier series 1968 Orthogonal expansions and their continuous analogues (Proc. Conf. Edwardsville, Ill 1967) pp. 235-255. Southern Illinois Univ. Press, Carbondale Ill.

[10] Lacey, M. , Thiele, C. A proof of boundedness of the Carleson operator Math. Res. Lett. 7[2000] pp. $361-370$.

[11] Muscalu, C. Tao, T. Thiele, C. A counterexample to an endpoint question of Christ and Kiselev to appear in Math. Res. Lett.

[12] Nazarov, F. and Treil, S., The hunt for a Bellman function: applications to estimates for singular integral operators and to other classical problems in harmonic analysis. (Russian) Algebra i Analiz 8 [1996] no 5. pp. 32-162. translation in St Petersburg Math J. 8 [1997] pp. 721-824.

[13] Sch'nol I. On the behaviour of the Schrödinger equation Mat. Sb. 42 [1957] pp.273-286 (Russian).

[14] Simon, B. Schrödinger semigroups, Bull. AMS 7 [1982] pp. 447-526.

[15] Thiele, C., Time-frequency analysis in the discrete phase plane PhD thesis, Yale 1995. Also in: Coifman R. (ed.) Selected Theses, World Scientific Publishing, Singapore [2000]

[16] Verblunsky, S., On positive harmonic functions Proc. London Math. Soc. 34 [1934] pp. 125-157.

[17] Verblunsky, S., On positive harmonic functions II Proc. London Math. Soc. 40 [1935] pp. 290-320.

[18] Zakharov, V.E., Shabat, A.B., A refined theory of two dimensional self-focussing and onedimensional self-modulation of waves in non-linear media Zh. Eksp. Teor. Fiz. 61 [1971] pp. 118-134; Soviet Physics JETP 34 [1972] pp. 62-69.

Department of Mathematics, UCla, Los Angeles CA 90095-1555

E-mail address: camil@math.ucla.edu

Department of Mathematics, UCla, Los Angeles CA 90095-1555

E-mail address: tao@math.ucla.edu

Department of Mathematics, UCLA, Los Angeles CA 90095-1555

E-mail address: thiele@math.ucla.edu 